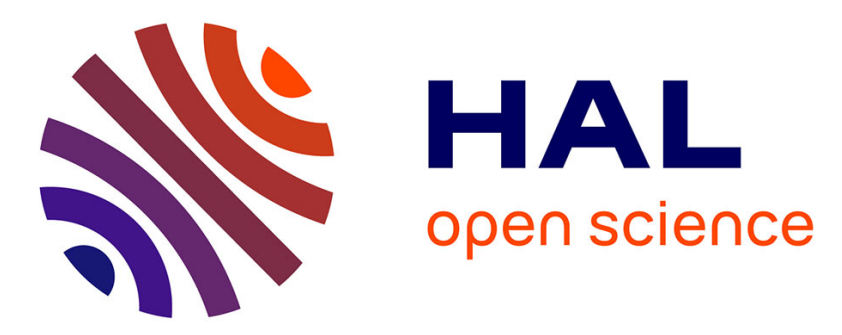

\title{
Kinetic-fluid derivation and mathematical analysis of cross-diffusion-Brinkman system
}

\author{
Mostafa Bendahmane, Fahd Karami, Mohammed Zagour
}

\section{To cite this version:}

Mostafa Bendahmane, Fahd Karami, Mohammed Zagour. Kinetic-fluid derivation and mathematical analysis of cross-diffusion-Brinkman system. Mathematical Methods in the Applied Sciences, 2019, 10.1002/mma.5139 . hal-01680584

\section{HAL Id: hal-01680584 \\ https://hal.inria.fr/hal-01680584}

Submitted on 30 Oct 2018

HAL is a multi-disciplinary open access archive for the deposit and dissemination of scientific research documents, whether they are published or not. The documents may come from teaching and research institutions in France or abroad, or from public or private research centers.
L'archive ouverte pluridisciplinaire $\mathbf{H A L}$, est destinée au dépôt et à la diffusion de documents scientifiques de niveau recherche, publiés ou non, émanant des établissements d'enseignement et de recherche français ou étrangers, des laboratoires publics ou privés. 


\title{
KINETIC-FLUID DERIVATION AND MATHEMATICAL ANALYSIS OF CROSS-DIFFUSION-BRINKMAN SYSTEM
}

\author{
M. BENDAHMANE, F. KARAMI AND M. ZAGOUR
}

\author{
Mostafa Bendahmane \\ Institut de Mathématiques de Bordeaux, \\ Université de Bordeaux, 33076 Bordeaux Cedex, France \\ FAHD Karami AND MOHAmed Zagour \\ École Suprieure de Technologie d'Essaouira, \\ Université Cadi Ayyad, Maroc
}

\begin{abstract}
In this paper, we propose a new nonlinear model describing dynamical interaction of two species within viscous flow. The proposed model is a cross-diffusion system coupled with Brinkman problem written in terms of velocity fluid, vorticity and pressure, and describing the flow patterns driven by an external source depending on the distribution of species. In the first part, we derive a macroscopic models from the kinetic-fluid equations by using the micro-macro decomposition method. Basing on Schauder fixed-point theory, we prove the existence of weak solutions for the derived model in the second part. The last part is devoted to develop a one dimensional finite volume approximation for the kinetic-fluid model, which are uniformly stable along the transition from kinetic to macroscopic regimes. Our computation method is validated with various numerical tests.
\end{abstract}

\section{INTRODUCTION}

During the last few decades, cross-diffusion systems have attracted a growth intention of mathematicians and biologists. Mainly for their ability to predict some interesting features in the studied field, for instance population dynamic in ecology. In mathematical biology applications, cross diffusion systems arise to model segregation phenomena between competing species. In real life, we observe that prey (for e.g. phytoplankton) has the tendency to keep away from predator (for e.g. zooplankton) at the same time predator has the tendency to get closer to the prey (see $[31,36,40]$ for more details). We observe also that many species live in complex flow so that species are transported in direction of the flow. Conversely, the velocity of the flow is under the influence of external forces including the density of species. Thus, it is interesting to study the dynamic of the interacting species on the basis of the coupled cross-diffusion-fluid model. In the following, we interest to the viscous flow in porous medium which is always modeled by Brinkman equations stating momentum and the conservation of mass of the fluid. We note that Brinkman problem is a parameter dependent combination of the Darcy and Stokes models, so that the flow is dominated by Darcy regime and by Stokes elsewhere. Motivated by this phenomena, in this paper we propose a nonlinear cross-diffusion system include additional terms accounting for the advection of each species with the fluid velocity, coupled with Brinkman problem written in terms of velocity fluid, vorticity and pressure, and describing the flow patterns driven by an external source depending on the distribution of species.

In order to state our problem, let $\Omega \subset \mathbb{R}^{3}$ be a simply connected, and bounded porous domain saturated with a Newtonian incompressible fluid, where also the two species are present. The

1991 Mathematics Subject Classification. Primary:

Key words and phrases. Asymptotic preserving scheme, kinetic theory, cross-diffusion, Brinkman flows, finite volume method. 
physical scenario of interest can be therefore described by a coupled system written in terms of the fluid velocity $\boldsymbol{u}$, the rescaled fluid vorticity $\boldsymbol{\omega}$, the fluid pressure $p$, and the densities of two species $c$ and $s$. The cross-diffusion-Brinkman model can be written for $(t, \boldsymbol{x})$ in $\Omega_{T}:=(0, T] \times \Omega$ :

$$
\left\{\begin{array}{l}
c_{t}+\boldsymbol{u} \cdot \nabla c-\Delta\left[\left(D_{c}(c)+a_{11} c+a_{12} s\right) c\right]=H_{c}(c, s), \\
s_{t}+\boldsymbol{u} \cdot \nabla s-\Delta\left[\left(D_{s}(s)+a_{21} c+a_{22} s\right) s\right]=H_{s}(c, s), \\
\mathbb{K}^{-1} \boldsymbol{u}+\sqrt{\mu} \operatorname{curl} \boldsymbol{\omega}+\nabla p=Q(c, s) \boldsymbol{g}+\mathbf{F}, \quad \boldsymbol{\omega}-\sqrt{\mu} \operatorname{curl} \boldsymbol{u}=\mathbf{0}, \quad \operatorname{div}_{x} \boldsymbol{u}=0,
\end{array}\right.
$$

where $D_{c}(c), D_{s}(s)$ are the nonlinear diffusivities functions and $a_{i j}>0$ for $i, j=1,2$ is known as self and cross-diffusion rates. The parameter $\mu$ is the fluid viscosity in the considered regime, it is assumed independent of the densities of species $c$ and $s, \mathbb{K}(\boldsymbol{x})$ is the permeability tensor rescaled with viscosity, $Q(c, s) \boldsymbol{g}$ represents the force exerted by the densities on the fluid motion, $\mathbf{F}(t, \boldsymbol{x})$ is an external force applied to the porous medium, the functions $H_{c}$ and $H_{s}$ are the reaction terms. A typical example of $H_{c}$ and $H_{s}$ is given by Lotka-Voltera (logistic) type growth term:

$$
\left\{\begin{array}{l}
H_{c}(c, s)=c\left(a_{1}-b_{1} c-d_{1} s\right), \\
H_{s}(c, s)=s\left(a_{2}-b_{2} c-d_{2} s\right),
\end{array}\right.
$$

where $a_{1}$ and $a_{2}$ are the Malthusian growth coefficients, and $b_{1}, d_{2}$ and $b_{2}, d_{1}$ are the coefficients of intra- and inter-species competition, respectively. Note that our system reads for suitably smooth functions $c$ and $s$ as follows

$$
\left\{\begin{array}{l}
c_{t}+\boldsymbol{u} \cdot \nabla c-\operatorname{div}\left[\left(D_{c}(c)+2 a_{11} c+a_{12} s\right) \nabla c+a_{12} c \nabla s\right]=H_{c}(c, s) \\
s_{t}+\boldsymbol{u} \cdot \nabla s-\operatorname{div}\left[\left(D_{s}(s)+2 a_{21} c+2 a_{22} s\right) \nabla s+a_{21} s \nabla c\right]=H_{s}(c, s), \quad \text { in } \Omega_{T} \\
\mathbb{K}^{-1} \boldsymbol{u}+\sqrt{\mu} \operatorname{curl} \boldsymbol{\omega}+\nabla p=Q(c, s) \boldsymbol{g}+\mathbf{F}, \quad \boldsymbol{\omega}-\sqrt{\mu} \operatorname{curl} \boldsymbol{u}=\mathbf{0}, \quad \operatorname{div}_{x} \boldsymbol{u}=0
\end{array}\right.
$$

Our model (1.3) is complemented with the following boundary condition and initial data:

$$
\begin{array}{r}
\left(c \boldsymbol{u}-\left(D_{c}(c)+2 a_{11} c+a_{12} s\right) \nabla c-a_{12} c \nabla s\right) \cdot \mathbf{n}=0, \\
\left(s \boldsymbol{u}-\left(D_{s}(s)+2 a_{21} c+2 a_{22} s\right) \nabla s-a_{21} s \nabla c\right) \cdot \mathbf{n}=0, \\
\boldsymbol{u} \cdot \mathbf{n}=u_{\partial}, \boldsymbol{\omega} \times \mathbf{n}=\boldsymbol{\omega}_{\partial},(t, \boldsymbol{x}) \in[0, T] \times \partial \Omega, \\
c(t=0, \boldsymbol{x})=c_{0}, \quad s(t=0, \boldsymbol{x})=s_{0}, \quad \boldsymbol{x} \in \Omega .
\end{array}
$$

Note that in the case $\left(a_{i, j}\right)_{1 \leq i, j \leq 2}:=0$, our model can be reduced to the recent model by [1], in which the authors proposed reaction-diffusion system representing the bacteria-chemical mass exchange, coupled with Brinkman problem.

To the best of our knowledge, recently only there are few papers proposing the augmented velocityvorticity-pressure formulation (augmented Brinkman model) without reaction-diffusion system coupling. It was initially proposed by [42], where the authors added vorticity as a new unknown variable. In $[4,5]$, the authors proposed the analysis of this model using mixed finite element method for standard and non-standard boundary conditions, respectively. Next in [35], the authors studied numerically an advection-diffusion-reaction system coupled with an incompressible viscous flow. When the fluid is at rest $(\boldsymbol{u}=0)$, several works have been proposed in the literature to investigate the theoretical and numerical analysis of the cross-diffusion model. For instance, the works by [15] and [43] include the analysis of the weak solution and the global existence of solution. Moreover, the authors in [41] specified the conditions for the existence of unstable equilibrium points. In the other hand, many numerical methods are proposed to approximate the solution. We refer the reader to finite difference method in [21], finite element method in [8], deterministic particle method in [29], finite volume method in [2,3,6] and positivity-preserving Euler-Galerkin method in [22]. We want to mention here that this is the first attemps to derive macroscopic model with nonlinear diffusitive terms. Moreover, in our contribution we present a new model (Cross-diffusion-Brinkman system) that combine interaction of the species in the presence of fluid.

In this paper, we derive a general cross-diffusion models coupled with the Brinkman problem from the kinetic-fluid using micro-macro decomposition method. In particular, we are interesting to derive our Cross-diffusion-Brinkman model (1.3). The idea of micro-macro decomposition is 
to write to unknown distribution function as a sum of an equilibrium and a deviation. We note that this method permits to reformulate the singularly perturbed kinetic system into an equivalent micro-macro formulation which is a regular perturbation of the derivative model. Thus solving numerically the equivalent micro-macro formulation instead of the perturbed kinetic system will permit to shift automatically to the limit problem, if the perturbation parameter (Knudsen parameter or sometimes it refers as mean free path ) is too small. Several contributions investigated the asymptotic limit: in the case of diffusion limit, see for instance [14, 17, 34], recently [23, 24] in the case of anomalous diffusion limit, hyperbolic model [38], Keller-Segel models of pattern formation in biological tissues $[9,12,18]$. Note that there are different approaches to construct such scheme for kinetic models in various contexts. For instance, the authors in [10,11] developed the approach of continuum mechanics based on micro-macro derivation in biological tissue and [13] for incompressible Navier-Stock. We refer the reader to this interesting overview [7]. An other approach largely studied based on domain decomposition (for linear transport equation, see for instance $[28,33])$.

The outline of this article is the following: In Section 2, we present the kinetic model and its proprieties. Next, we perform the micro-macro formulation, which is the key ingredient in the construction of our numerical method. Section 3 is devoted to proving existence of weak solution for derived macroscopic model. Our numerical method is demonstrated in Section 4 with various numerical tests.

\section{Derivation of CROSS-DIFFUSION-BRINKMAN MODELS}

This section aims to derive general macroscopic models using micro-macro decomposition method following the line of the paper [9]. Note that the authors in [9] have derived a macroscopic models of Keller-Segel type, which describe the chemotaxis phenomenon [32]. The first subsection is devoted to present the proprieties of the kinetic system which lead to an equivalent micro-macro formulation. We will formally derive a general macroscopic models of type cross-diffusion-Brinkman system in the second subsection, while the last subsection is devoted to deriving our model (1.3) as a particular model. We want to mention here that the novelty in this first part is that we derive the cross-diffusion system with nonlinear diffusitive functions coupled with Brinkman problem.

2.1. The kinetic-fluid model. In order to derive a general macroscopic cross-diffusion-Brinkman model from the kinetic model, we consider the parabolic-parabolic scaling. Thus, the kinetic-fluid model is as follows

$$
\left\{\begin{array}{l}
\left(\varepsilon \partial_{t}+\xi \cdot \nabla_{x}\right) f_{1}^{\varepsilon}=\frac{1}{\varepsilon} \mathcal{T}_{1}\left[f_{2}^{\varepsilon}\right]\left(f_{1}^{\varepsilon}\right)+G_{1}\left(f_{1}^{\varepsilon}, f_{2}^{\varepsilon}, \boldsymbol{u}, \xi\right), \\
\left(\varepsilon \partial_{t}+\xi \cdot \nabla_{x}\right) f_{2}^{\varepsilon}=\frac{1}{\varepsilon} \mathcal{T}_{2}\left[f_{1}^{\varepsilon}\right]\left(f_{2}^{\varepsilon}\right)+G_{2}\left(f_{1}^{\varepsilon}, f_{2}^{\varepsilon}, \boldsymbol{u}, \xi\right), \\
\mathbb{K}^{-1} \boldsymbol{u}+\sqrt{\mu} \operatorname{curl} \boldsymbol{\omega}+\nabla p=Q\left(\int_{V} f_{1}^{\varepsilon} d \xi, \int_{V} f_{2}^{\varepsilon} d \xi\right) \boldsymbol{g}+\mathbf{F}, \boldsymbol{\omega}-\sqrt{\mu} \operatorname{curl} \boldsymbol{u}=\mathbf{0}, \operatorname{div} \boldsymbol{u}=0
\end{array}\right.
$$

where $f_{1}, f_{2}$ are the generalized distribution functions which depend on time $t$, position $x \in \Omega \subset \mathbb{R}^{d}$ and velocity $\xi \in V \subset \mathbb{R}^{d}$ ( $V$ assumed to be bounded and symmetric). The remains macroscopic variables and parameters, namely $\boldsymbol{u}, \mu, \boldsymbol{\omega}, p, Q, \boldsymbol{g}$, and $\mathbf{F}$, are defined in the introduction. The Knudsen parameter $\varepsilon$ measures the distance of the system to its equilibrium. Specifically, when $\varepsilon$ is small, the system is close to an equilibrium state, while for large $\varepsilon$, the system is far from equilibrium. Moreover, $\mathcal{T}_{1}\left[f_{2}\right], \mathcal{T}_{2}\left[f_{1}\right]$ and $G_{i}, i=1,2$ are, respectively, the turning and interaction operators, assumed to satisfy the following:

- The turning operators are decomposed as follows

$$
\mathcal{T}_{1}\left[f_{2}\right](h)=\mathcal{T}_{1}^{1}(h)+\mathcal{T}_{1}^{2}\left[f_{2}\right](h), \quad \mathcal{T}_{2}\left[f_{1}\right](h)=\mathcal{T}_{1}^{2}(h)+\mathcal{T}_{2}^{2}\left[f_{1}\right](h),
$$


where $\mathcal{T}_{j}^{i}$ for $i, j=1,2$ are given by

$$
\mathcal{T}_{j}^{i}(h)=\int_{V}\left[T_{j}^{i}\left(\xi^{*}, \xi\right) h\left(t, x, \xi^{*}\right)-T_{j}^{i}\left(\xi, \xi^{*}\right) h(t, x, \xi)\right] d \xi^{*},
$$

with $T_{j}^{i}\left(\xi, \xi^{*}\right)$ is the probability kernel for the new velocity $\xi \in V$, given that the previous velocity was $\xi^{*}$. The dependence on $f_{2}\left(\operatorname{resp} . f_{1}\right)$ of the operator $\mathcal{T}_{1}^{2}[f 2]\left(\operatorname{resp} . \mathcal{T}_{2}^{2}[f 1]\right)$ stems from $T_{2}^{1}$ ( resp. $T_{2}^{2}$ ). We assume that $\mathcal{T}_{1}^{1}$ is independent on $f_{2}$ and $\mathcal{T}_{2}^{1}$ is independent on $f_{1}$. In sequence, we shall consider $\mathcal{T}_{1}^{1}(h)=\mathcal{L}_{1}(h)$ and $\mathcal{T}_{2}^{1}(h)=\mathcal{L}_{2}(h)$.

- We assume that

$$
\int_{V} \mathcal{L}_{1}(h) d \xi=\int_{V} \mathcal{T}_{1}^{2}\left[f_{2}\right](h) d \xi=\int_{V} \mathcal{L}_{2}(h) d \xi=\int_{V} \mathcal{T}_{2}^{2}\left[f_{1}\right](h) d \xi=0 .
$$

- There exists a bounded velocity distribution $M_{i}(\xi)>0,(i=1,2)$ independent of $t$ and $x$, such that

$$
T_{1}^{1}\left(\xi, \xi^{*}\right) M_{1}\left(\xi^{*}\right)=T_{1}^{1}\left(\xi^{*}, \xi\right) M_{1}(\xi), \quad T_{2}^{1}\left(\xi, \xi^{*}\right) M_{2}\left(\xi^{*}\right)=T_{2}^{1}\left(\xi^{*}, \xi\right) M_{2}(\xi)
$$

holds. Furthermore, $M_{i}$ are normalized and the flow produced by these equilibrium distributions vanishes

$$
\int_{V} M_{i}(\xi) d \xi=1, \quad \int_{V} \xi M_{i}(\xi) d \xi=0, \quad i=1,2 .
$$

The probability kernels $T_{1}^{1}\left(\xi, \xi^{*}\right)$ and $T_{2}^{1}\left(\xi, \xi^{*}\right)$ are bounded, and there exists a constant $\sigma_{i}>0, i=1,2$, such that

$$
T_{1}^{1}\left(\xi, \xi^{*}\right) \geq \sigma_{1} M_{1}(\xi), \quad T_{2}^{1}\left(\xi, \xi^{*}\right) \geq \sigma_{2} M_{2}(\xi)
$$

for all $\left(\xi, \xi^{*}\right) \in V \times V$.

- Moreover, we assume that the interaction operator $G_{i}(i=1,2)$ satisfies the following:

$$
G_{i}\left(f_{1}, f_{2}, \boldsymbol{u}, \xi\right)=G_{i 1}\left(f_{1}, f_{2}, \boldsymbol{u}, \xi\right)+\varepsilon G_{i 2}\left(f_{1}, f_{2}, \xi\right),
$$

where

$$
\int_{V} G_{i 1}\left(f_{1}, f_{2}, \boldsymbol{u}, \xi\right) d \xi=0
$$

for $i=1,2$.

Thanks to technical calculations in [20], the operator $\mathcal{L}_{i}(i=1,2)$ has the following proprieties (the proof of the following lemma can be found in [9]):

Lemma 2.1. Assume that the hypothesis (2.4), (2.5) and (2.6) hold. Then, the following properties of the operators $\mathcal{L}_{1}$ and $\mathcal{L}_{2}$ hold true:

1) The operator $\mathcal{L}_{i}$ is self-adjoint in the space $\mathrm{L}^{2}\left(V, \frac{d \xi}{M_{i}}\right)$.

2) For $h \in \mathrm{L}^{2}$, the equation $\mathcal{L}_{i}(g)=h,(i=1,2)$ has a unique solution $g \in \mathrm{L}^{2}\left(V, \frac{d \xi}{M_{i}}\right)$, satisfying

$$
\int_{V} g(\xi) d \xi=0 \quad \text { if and only if } \quad \int_{V} h(\xi) d \xi=0 .
$$

3) The equation $\mathcal{L}_{i}(g)=\xi M_{i}(\xi)$, has a unique solution will be denoted by $\theta_{i}(\xi)$, for $i=1,2$.

4) The kernel of $\mathcal{L}_{i}$ is $N\left(\mathcal{L}_{i}\right)=\operatorname{vect}\left(M_{i}(\xi)\right), i=1,2$. 
2.2. The equivalent micro-macro formulation. In this subsection, we rewrite each kinetic equation in (2.1) as an equivalent system coupling a hydrodynamic part with a kinetic part. For that we decompose $f_{i},(i=1,2)$ into a main part that is close to the equilibrium in diffusive regimes, and another part that vanishes in this limit, i.e

$$
f_{1}(t, x, \xi)=M_{1}(\xi) c(t, x)+\varepsilon g_{1}(t, x, \xi), \quad f_{2}(t, x, \xi)=M_{2}(\xi) s(t, x)+\varepsilon g_{2}(t, x, \xi),
$$

where

$$
c(t, x)=\int_{V} f_{1}(t, x, \xi) d \xi, \quad s(t, x)=\int_{V} f_{2}(t, x, \xi) d \xi
$$

We recall that $\left\langle g_{i}\right\rangle:=\int_{V} g_{i}(t, x, \xi) d \xi=0$ for $i=1,2$. Inserting $f_{1}$ and $f_{2}$ in the kinetic model (2.1) and using the proprieties of the kernel operators, we get

$$
\begin{array}{r}
\partial_{t}\left(M_{1} c\right)+\varepsilon \partial_{t} g_{1}+\frac{1}{\varepsilon} \xi M_{1} \cdot \nabla_{x} c+\xi \cdot \nabla_{x} g_{1}=\frac{1}{\varepsilon} \mathcal{L}_{1}\left(g_{1}\right)+\frac{1}{\varepsilon} \mathcal{T}_{1}^{2}\left[f_{2}\right]\left(M_{1}(\xi) c\right) \\
+\mathcal{T}_{1}^{2}\left[f_{2}\right]\left(g_{1}\right)+\frac{1}{\varepsilon} G_{11}\left(f_{1}, f_{2}, \boldsymbol{u}, \xi\right)+G_{12}\left(f_{1}, f_{2}, \xi\right), \\
\partial_{t}\left(M_{2} s\right)+\varepsilon \partial_{t} g_{2}+\frac{1}{\varepsilon} \xi M_{2} \cdot \nabla_{x} s+\xi \cdot \nabla_{x} g_{2}=\frac{1}{\varepsilon} \mathcal{L}_{2}\left(g_{2}\right)+\frac{1}{\varepsilon} \mathcal{T}_{2}^{2}\left[f_{1}\right]\left(M_{2}(\xi) s\right) \\
+\mathcal{T}_{2}^{2}\left[f_{1}\right]\left(g_{2}\right)+\frac{1}{\varepsilon} G_{21}\left(f_{1}, f_{2}, \boldsymbol{u}, \xi\right)+G_{22}\left(f_{1}, f_{2}, \xi\right), \\
\mathbb{K}^{-1} \boldsymbol{u}+\sqrt{\mu} \operatorname{curl} \boldsymbol{\omega}+\nabla p=Q(c, s) \boldsymbol{g}+\mathbf{F}, \quad \boldsymbol{\omega}-\sqrt{\mu} \mathbf{c u r l} \boldsymbol{u}=\mathbf{0}, \quad \operatorname{div} \boldsymbol{u}=0 .
\end{array}
$$

The micro-macro formulation equivalent to system (2.1) is obtained by two steps. Firstly, we use a projection technique to separate the macroscopic densities $(c(t, x), s(t, x))$ and microscopic quantities $\left(g_{1}(t, x, \xi), g_{2}(t, x, \xi)\right)$. For that, let $P_{M_{i}}$, denote the orthogonal projection onto $N\left(\mathcal{L}_{i}\right)$, for $i=1,2$. It follows

$$
P_{M_{i}}(h)=\langle h\rangle M_{i}, \quad \text { for any } h \in \mathrm{L}^{2}\left(V, \frac{d \xi}{M_{i}}\right),
$$

for $i=1,2$. Regarding the orthogonal projections $P_{M_{1}}, P_{M_{2}}$, we have the following result:

Lemma 2.2. (cf. [9]) For the projection $P_{M_{1}}, P_{M_{2}}$, we have the following properties :

$$
\begin{gathered}
\left(I-P_{M_{1}}\right)\left(M_{1} c\right)=\left(I-P_{M_{2}}\right)\left(M_{2} s\right)=P_{M_{1}}\left(g_{1}\right)=P_{M_{2}}\left(g_{2}\right)=0, \\
\left(I-P_{M_{1}}\right)\left(\xi M_{1} \cdot \nabla_{x} c\right)=\xi M_{1} \cdot \nabla_{x} c, \quad\left(I-P_{M_{2}}\right)\left(\xi M_{2} \cdot \nabla_{x} s\right)=\xi M_{2} \cdot \nabla_{x} s, \\
\left(I-P_{M_{1}}\right)\left(\mathcal{T}_{1}^{2}\left[f_{2}\right]\left(M_{1}(\xi) c\right)=\mathcal{T}_{1}^{2}\left[f_{2}\right]\left(M_{1}(\xi) c\right), \quad\left(I-P_{M_{2}}\right)\left(\mathcal{T}_{2}^{2}\left[f_{1}\right]\left(M_{2}(\xi) s\right)\right)=\mathcal{T}_{2}^{2}\left[f_{1}\right]\left(M_{2}(\xi) s\right),\right. \\
\left(I-P_{M_{2}}\right)\left(\mathcal{T}_{2}^{2}\left[f_{1}\right](h)\right)=\mathcal{T}_{2}^{2}\left[f_{1}\right](h), \quad\left(I-P_{M_{1}}\right)\left(\mathcal{T}_{1}^{2}\left[f_{2}\right](h)\right)=\mathcal{T}_{1}^{2}\left[f_{2}\right](h), \\
\left(I-P_{M_{1}}\right)\left(\mathcal{L}_{i}(h)\right)=\mathcal{L}_{i}(h), \quad\left(I-P_{M_{i}}\right)\left(G_{i 1}\left(f_{1}, f_{2}, \boldsymbol{u}, \xi\right)\right)=G_{i 1}\left(f_{1}, f_{2}, \boldsymbol{u}, \xi\right) \quad \text { for } i=1,2 .
\end{gathered}
$$

Taking the operators $I-P_{M_{1}}$ and $I-P_{M_{2}}$ into (2.10) and (2.11), respectively, and using Lemma 2.2, yield the following microscopic equations:

$$
\begin{array}{r}
\varepsilon \partial_{t} g_{1}+\frac{1}{\varepsilon} \xi M_{1} \cdot \nabla_{x} c+\left(I-P_{M_{1}}\right)\left(\xi \cdot \nabla_{x} g_{1}\right)=\frac{1}{\varepsilon} \mathcal{L}_{1}\left(g_{1}\right)+\frac{1}{\varepsilon} \mathcal{T}_{1}^{2}\left[f_{2}\right]\left(M_{1}(\xi) c\right) \\
+\mathcal{T}_{1}^{2}\left[f_{2}\right]\left(g_{1}\right)+\frac{1}{\varepsilon} G_{11}\left(f_{1}, f_{2}, \boldsymbol{u}, \xi\right)+\left(I-P_{M_{1}}\right) G_{12}\left(f_{1}, f_{2}, \xi\right), \\
\varepsilon \partial_{t} g_{2}+\frac{1}{\varepsilon} \xi M_{2} \cdot \nabla_{x} s+\left(I-P_{M_{2}}\right)\left(\xi \cdot \nabla_{x} g_{2}\right)=\frac{1}{\varepsilon} \mathcal{L}_{2}\left(g_{2}\right)+\frac{1}{\varepsilon} \mathcal{T}_{2}^{2}\left[f_{1}\right]\left(M_{2}(\xi) s\right) \\
+\mathcal{T}_{2}^{2}\left[f_{1}\right]\left(g_{2}\right)+\frac{1}{\varepsilon} G_{21}\left(f_{1}, f_{2}, \boldsymbol{u}, \xi\right)+\left(I-P_{M_{1}}\right) G_{22}\left(f_{1}, f_{2}, \xi\right) .
\end{array}
$$


Now, integrating (2.11) and (2.10) over $\xi$ and using (2.4)-(2.9), we obtain the following hydrodynamic equations:

$$
\begin{aligned}
& \partial_{t} c+\left\langle\xi \cdot \nabla_{x} g_{1}\right\rangle=\left\langle G_{12}\left(f_{1}, f_{2}, \xi\right)\right\rangle, \\
& \partial_{t} s+\left\langle\xi \cdot \nabla_{x} g_{2}\right\rangle=\left\langle G_{22}\left(f_{1}, f_{2}, \xi\right)\right\rangle .
\end{aligned}
$$

Finally, thanks to $(2.13),(2.14),(2.15)$, and (2.16), the micro-macro formulation reads

$$
\left\{\begin{aligned}
\varepsilon \partial_{t} g_{1}+\frac{1}{\varepsilon} \xi M_{1} \cdot \nabla_{x} c & +\left(I-P_{M_{1}}\right)\left(\xi \cdot \nabla_{x} g_{1}\right)=\frac{1}{\varepsilon} \mathcal{L}_{1}\left(g_{1}\right) \\
+ & \frac{1}{\varepsilon} \mathcal{T}_{1}^{2}\left[f_{2}\right]\left(M_{1}(\xi) c\right)+\mathcal{T}_{1}^{2}\left[f_{2}\right]\left(g_{1}\right) \\
+ & \frac{1}{\varepsilon} G_{11}\left(f_{1}, f_{2}, \boldsymbol{u}, \xi\right)+\left(I-P_{M_{1}}\right) G_{12}\left(f_{1}, f_{2}, \xi\right), \\
\partial_{t} c+\left\langle\xi \cdot \nabla_{x} g_{1}\right\rangle= & \left\langle G_{12}\left(f_{1}, f_{2}, \xi\right)\right\rangle \\
\varepsilon \partial_{t} g_{2}+\frac{1}{\varepsilon} \xi M_{2} \cdot \nabla_{x} s+ & \left(I-P_{M_{2}}\right)\left(\xi \cdot \nabla_{x} g_{2}\right)=\frac{1}{\varepsilon} \mathcal{L}_{2}\left(g_{2}\right) \\
& +\frac{1}{\varepsilon} \mathcal{T}_{2}^{2}\left[f_{1}\right]\left(M_{2}(\xi) s\right)+\mathcal{T}_{2}^{2}\left[f_{1}\right]\left(g_{2}\right) \\
& +\frac{1}{\varepsilon} G_{21}\left(f_{1}, f_{2}, \boldsymbol{u}, \xi\right)+\left(I-P_{M_{2}}\right) G_{22}\left(f_{1}, f_{2}, \xi\right), \\
\partial_{t} s+\left\langle\xi \cdot \nabla_{x} g_{2}\right\rangle= & \left\langle G_{22}\left(f_{1}, f_{2}, \xi\right)\right\rangle, \\
\mathbb{K}^{-1} \boldsymbol{u}+\sqrt{\mu} \mathbf{c u r l} \boldsymbol{\omega}+ & \nabla p=Q(c, s) \boldsymbol{g}+\mathbf{F}, \quad \boldsymbol{\omega}-\sqrt{\mu} \mathbf{c u r l} \boldsymbol{u}=\mathbf{0}, \quad \operatorname{div} \boldsymbol{u}=0
\end{aligned}\right.
$$

Note that system (2.17) is the micro-macro formulation of the kinetic-fluid model (2.1). The following proposition shows that system (2.17) and the model (2.1) are equivalents.

Proposition 2.1. i) Let $\left(f_{1}, f_{2}, \boldsymbol{u}, \boldsymbol{\omega}, p\right)$ be a solution of the kinetic system (2.1). Then the functions $\left(c, g_{1}, s, g_{2}, \boldsymbol{u}, \boldsymbol{\omega}, p\right)$ (where $c=\left\langle f_{1}\right\rangle, s=\left\langle f_{2}\right\rangle, g_{1}=\frac{1}{\varepsilon}\left(f_{1}-M_{1} c\right), g_{2}=\frac{1}{\varepsilon}\left(f_{2}-M_{2} s\right)$ ) is a solution to a coupled system (2.17) with the associated initial data

$$
\begin{aligned}
& c(t=0)=c_{0}=\left\langle f_{10}\right\rangle, \quad g_{1}(t=0)=g_{10}=\frac{1}{\varepsilon}\left(f_{10}-M_{1} c_{0}\right), \\
& s(t=0)=s_{0}=\left\langle f_{20}\right\rangle, \quad g_{2}(t=0)=g_{20}=\frac{1}{\varepsilon}\left(f_{20}-M_{2} s_{0}\right) .
\end{aligned}
$$

ii) Conversely, if $\left(c, g_{1}, s, g_{2}, \boldsymbol{u}, \boldsymbol{\omega}, p\right)$ satisfies system (2.17) with initial data $\left(c_{0}, g_{10}, s_{0}, g_{20}\right)$ such that $\left\langle g_{10}\right\rangle=\left\langle g_{20}\right\rangle=0$, then $\left(f_{1}=M_{1} c+\varepsilon g_{1}, f_{2}=M_{2} s+\varepsilon g_{2}, \boldsymbol{u}, \boldsymbol{\omega}, p\right)$ is a solution to kinetic model (2.1) with initial data $f_{10}=M_{1} c_{0}+\varepsilon g_{10}, f_{20}=M_{2} s_{0}+\varepsilon g_{20}$ and we have $c=\left\langle f_{1}\right\rangle, s=\left\langle f_{2}\right\rangle$ and $\left\langle g_{1}\right\rangle=\left\langle g_{2}\right\rangle=0$.

Remark 2.1. The proof of $i)$ is already detailed above. For the proof of ii) we refer the reader to proof of Theorem 1 in [9].

In order to develop asymptotic analysis of system (2.17), we assume that the turning operators $\mathcal{T}_{1}^{2}, \mathcal{T}_{2}^{2}$ and the interactions terms $G_{i 1}, G_{i 2}$ satisfy the following asymptotic behavior $\varepsilon \rightarrow 0$ : 


$$
\mathcal{T}_{1}^{2}\left[M_{2}(\xi) s+\varepsilon g_{2}\right]=\mathcal{T}_{1}^{2}\left[M_{2}(\xi) s\right]+O(\varepsilon), \quad \mathcal{T}_{2}^{2}\left[M_{1}(\xi) c+\varepsilon g_{1}\right]=\mathcal{T}_{2}^{2}\left[M_{1}(\xi) c\right]+O(\varepsilon),
$$

and for $i=1,2$,

$$
\begin{aligned}
G_{i 1}\left(M_{1}(\xi) c+\varepsilon g_{1}, M_{2}(\xi) s+\varepsilon g_{2}, \boldsymbol{u}, \xi\right) & =G_{i 1}\left(M_{1}(\xi) c, M_{2}(\xi) s, \boldsymbol{u}, \xi\right)+O(\varepsilon), \\
G_{2 i}\left(M_{1}(\xi) c+\varepsilon g_{1}, M_{2}(\xi) s+\varepsilon g_{2}, \xi\right) & =G_{2 i}\left(M_{1}(\xi) c, M_{2}(\xi) s, \xi\right)+O(\varepsilon) .
\end{aligned}
$$

2.3. Derivation of macroscopic models. This subsection is devoted to derive a macroscopic models from the micro-macro formulation (2.17) of the kinetic-fluid system (2.1) as $\varepsilon$ goes to 0 . First, we use (2.20)-(2.22) and (2.17), to obtain

$$
\begin{aligned}
& \mathcal{L}_{1}\left(g_{1}\right)=\xi M_{1} \cdot \nabla_{x} c-\mathcal{T}_{1}^{2}\left[M_{2}(\xi) s\right]\left(M_{1}(\xi) c\right)-G_{11}\left(M_{1} c, M_{2} s, \boldsymbol{u}, \xi\right)+O(\varepsilon), \\
& \mathcal{L}_{2}\left(g_{2}\right)=\xi M_{2} \cdot \nabla_{x} s-\mathcal{T}_{2}^{2}\left[M_{1}(\xi) c\right]\left(M_{2}(\xi) s\right)-G_{21}\left(M_{1} c, M_{2} s, \boldsymbol{u}, \xi\right)+O(\varepsilon) .
\end{aligned}
$$

Moreover from (2.4) and (2.9), yields the following computations:

$$
\begin{aligned}
g_{1}= & \left(\mathcal{L}_{1}\right)^{-1}\left(\xi M_{1} \cdot \nabla_{x} v\right)-\left(\mathcal{L}_{1}\right)^{-1}\left(\mathcal{T}_{1}^{2}\left[M_{2}(\xi) s\right]\left(M_{1}(\xi) c\right)\right) \\
& -\left(\mathcal{L}_{1}\right)^{-1}\left(G_{11}\left(M_{1} c, M_{2} s, \boldsymbol{u}, \xi\right)\right)+O(\varepsilon),
\end{aligned}
$$

and

$$
\begin{aligned}
g_{2}= & \left(\mathcal{L}_{2}\right)^{-1}\left(\xi M_{2} \cdot \nabla_{x} s\right)-\left(\mathcal{L}_{2}\right)^{-1}\left(\mathcal{T}_{2}^{2}\left[M_{1}(\xi) c\right]\left(M_{2}(\xi) s\right)\right) \\
& -\left(\mathcal{L}_{2}\right)^{-1}\left(G_{21}\left(M_{1} c, M_{2} s, \boldsymbol{u}, \xi\right)\right)+O(\varepsilon) .
\end{aligned}
$$

Inserting (2.23) and (2.24) into the second and the fourth equations in (2.17), yield the following coupled macroscopic model:

$$
\left\{\begin{array}{c}
\partial_{t} c+\left\langle\xi \cdot \nabla_{x}\left(\mathcal{L}_{1}\right)^{-1}\left(\xi M_{1} \cdot \nabla_{x} c\right)\right\rangle-\left\langle\xi \cdot \nabla_{x}\left(\mathcal{L}_{1}\right)^{-1}\left(\mathcal{T}_{1}^{2}\left[M_{2}(\xi) s\right]\left(M_{1}(\xi) c\right)\right)\right\rangle \\
-\left\langle\xi \cdot \nabla_{x}\left(\mathcal{L}_{1}\right)^{-1}\left(G_{11}\left(M_{1} c, M_{2} s, \boldsymbol{u}, \xi\right)\right)\right\rangle=\left\langle G_{12}\left(M_{1} c, M_{2} s, \xi\right)\right\rangle+O(\varepsilon), \\
\partial_{t} s+\left\langle\xi \cdot \nabla_{x}\left(\mathcal{L}_{2}\right)^{-1}\left(\xi M_{2} \cdot \nabla_{x} s\right)\right\rangle-\left\langle\xi \cdot \nabla_{x}\left(\mathcal{L}_{2}\right)^{-1}\left(\mathcal{T}_{2}^{2}\left[M_{1}(\xi) c\right]\left(M_{2}(\xi) s\right)\right)\right\rangle \\
-\left\langle\xi \cdot \nabla_{x}\left(\mathcal{L}_{2}\right)^{-1}\left(G_{21}\left(M_{1} c, M_{2} s, \boldsymbol{u}, \xi\right)\right)\right\rangle=\left\langle G_{22}\left(M_{1} c, M_{2} s, \xi\right)\right\rangle+O(\varepsilon), \\
\mathbb{K}^{-1} \boldsymbol{u}+\sqrt{\mu} \operatorname{curl} \boldsymbol{\omega}+\nabla p=Q(c, s) \boldsymbol{g}+\mathbf{F}, \quad \boldsymbol{\omega}-\sqrt{\mu} \operatorname{curl} \boldsymbol{u}=\mathbf{0}, \quad \operatorname{div} \boldsymbol{u}=0 .
\end{array}\right.
$$

The following lemma gives the calculations of the terms with the inverse of the operators $\mathcal{L}_{i}$ appearing in system (2.25), for $i=1,2$.

Lemma 2.3. (cf. [9]) Assume that the operators $\mathcal{L}_{1}, \mathcal{L}_{2}, G_{11}$ and $G_{21}$ are satisfy the assumptions above. Then we have the following identities:

$$
\begin{gathered}
\left\langle\xi \cdot \nabla_{x}\left(\mathcal{L}_{1}\right)^{-1}\left(\xi M_{1} \cdot \nabla_{x} c\right)\right\rangle=\operatorname{div}_{x}\left(\left\langle\xi \otimes \theta_{1}(\xi)\right\rangle \cdot \nabla_{x} c\right), \\
\left\langle\xi \cdot \nabla_{x}\left(\mathcal{L}_{2}\right)^{-1}\left(\xi M_{2} \cdot \nabla_{x} s\right)\right\rangle=\operatorname{div}_{x}\left(\left\langle\xi \otimes \theta_{2}(\xi)\right\rangle \cdot \nabla_{x} s\right), \\
\left\langle\xi \cdot \nabla_{x}\left(\mathcal{L}_{1}\right)^{-1}\left(\mathcal{T}_{1}^{2}\left[M_{2}(\xi) s\right]\left(M_{1}(\xi) c\right)\right)\right\rangle=\operatorname{div}_{x}\left\langle\frac{\theta_{1}(\xi)}{M_{1}(\xi)} c \mathcal{T}_{1}^{2}\left[M_{2}(\xi) s\right]\left(M_{1}(\xi)\right)\right\rangle, \\
\left\langle\xi . \nabla_{x}\left(\mathcal{L}_{i}\right)^{-1}\left(G_{i 1}\left(M_{1} c, M_{2} s, \boldsymbol{u}, \xi\right)\right)\right\rangle=\operatorname{div}_{x}\left\langle\frac{\theta_{i}(\xi)}{M_{i}(\xi), \xi} G_{i 1}\left(M_{1} c, M_{2} s, \boldsymbol{u}, \xi\right)\right\rangle, \quad i=1,2,
\end{gathered}
$$

In addition, in this paper we need the following identity

$$
\left\langle\xi . \nabla_{x}\left(\mathcal{L}_{2}\right)^{-1}\left(\mathcal{T}_{2}^{2}\left[M_{1}(\xi) c\right]\left(M_{2}(\xi) s\right)\right)\right\rangle=\operatorname{div}_{x}\left\langle\frac{\theta_{2}(\xi)}{M_{2}(\xi)} s \mathcal{T}_{2}^{2}\left[M_{1}(\xi) c\right]\left(M_{2}(\xi)\right)\right\rangle,
$$

where $\theta_{1}$ and $\theta_{2}$ are given in Lemma 2.1. 
Finally, thanks to system (2.25) and Lemma 2.3 we get the following macroscopic model:

$$
\left\{\begin{array}{l}
\partial_{t} c+\operatorname{div}_{x}\left(c \alpha_{1}(s)+\Gamma_{1}(c, s, \boldsymbol{u})-d_{c} \cdot \nabla_{x} c\right)-H_{1}(c, s)+O(\varepsilon)=0, \\
\partial_{t} s+\operatorname{div}_{x}\left(s \alpha_{2}(c)+\Gamma_{2}(c, s, \boldsymbol{u})-d_{s} \cdot \nabla_{x} s\right)-H_{2}(c, s)+O(\varepsilon)=0, \\
\mathbb{K}^{-1} \boldsymbol{u}+\sqrt{\mu} \operatorname{curl} \boldsymbol{\omega}+\nabla p=Q(c, s) \boldsymbol{g}+\mathbf{F}, \quad \boldsymbol{\omega}-\sqrt{\mu} \operatorname{curl} \boldsymbol{u}=\mathbf{0}, \quad \operatorname{div} \boldsymbol{u}=0,
\end{array}\right.
$$

where $d_{c}, d_{s}, \alpha_{1}(s), \alpha_{2}(c), \Gamma_{1}(c, s, \boldsymbol{u}), \Gamma_{2}(c, s, \boldsymbol{u}), H_{1}(c, s)$ and $H_{2}(c, s)$ are given by

$$
\begin{gathered}
d_{c}=-\int_{V} \xi \otimes \theta_{1}(\xi) d \xi, \quad d_{s}=-\int_{V} \xi \otimes \theta_{2}(\xi) d \xi \\
\alpha_{1}(s)=-\int_{V} \frac{\theta_{1}(\xi)}{M_{1}(\xi)} \mathcal{T}_{1}^{2}\left[M_{2}(\xi) s\right]\left(M_{1}(\xi)\right) d \xi, \quad \alpha_{2}(c)=-\int_{V} \frac{\theta_{2}(\xi)}{M_{2}(\xi)} \mathcal{T}_{2}^{2}\left[M_{1}(\xi) c\right]\left(M_{2}(\xi)\right) d \xi \\
\Gamma_{i}(c, s, \boldsymbol{u})=-\int_{V} \frac{\theta_{i}(\xi)}{M_{i}(\xi)} G_{i 1}\left(M_{1} c, M_{2} s, \boldsymbol{u}, \xi\right) d \xi, \quad i=1,2 \\
H_{1}(c, s)=\int_{V} G_{12}\left(M_{1} c, M_{2} s, \xi\right) d \xi, \quad H_{2}(c, s)=\int_{V} G_{22}\left(M_{1} c, M_{2} s, \xi\right) d \xi
\end{gathered}
$$

2.4. Derivation of cross-diffusion-Brinkman model. We consider the case where the set for velocity is the sphere of radius $r>0, V=r S^{d-1}$ and $x \in \Omega \subset \mathbb{R}^{2}$. We assume that the probability kernel $T_{i}^{1}$ is given by

This implies

$$
T_{i}^{1}=\frac{\sigma_{i}}{|V|}, \text { for } i=1,2
$$

$$
\mathcal{L}_{i}(f)=-\frac{\sigma_{i}}{|V|}(f|V|-\langle f\rangle) \text {, for } i=1,2 .
$$

In particular $M_{i}(\xi)=\frac{1}{|V|}$ satisfies the assumption (2.6) and $\theta_{i}$ is a solution of $\mathcal{L}_{i}\left(\theta_{i}(\xi)\right)=\xi M_{i}(\xi)$, where $\theta_{i}(\xi)=-\frac{\xi}{\sigma_{i}|V|}$, for $i=1,2$. We let $T_{1}^{2}[g]$ and $T_{2}^{2}[g]$ such that

$$
T_{1}^{2}[g]=a_{12} \frac{\beta_{1}}{|V|} \xi \cdot \nabla_{x} g \quad \text { and } \quad T_{2}^{2}[g]=a_{21} \frac{\beta_{2}}{|V|} \xi . \nabla_{x} g,
$$

where $\beta_{1}=\frac{\sigma_{1}}{r^{2}} d|V|$ and $\beta_{2}=\frac{\sigma_{2}}{r^{2}} d|V|$. It follows

$$
\mathcal{T}_{1}^{2}\left[M_{2} s\right]\left(M_{1}\right)=-a_{12} \frac{\beta_{1} M_{2}}{|V|^{2}} \xi \cdot \nabla_{x} s \quad \text { and } \quad \mathcal{T}_{2}^{2}\left[M_{1} c\right]\left(M_{2}\right)=-a_{21} \frac{\beta_{2} M_{1}}{|V|^{2}} \xi \cdot \nabla_{x} c .
$$

Therefore, we get

and

$$
\alpha_{1}(s)=-\frac{\beta_{1} a_{12}}{\sigma_{1}|V|^{2}}\left(\int_{V} \xi \otimes \xi d \xi\right) \cdot \nabla_{x} s,=-a_{12} \nabla_{x} s
$$

$$
\alpha_{2}(v)=-\frac{\beta_{2} a_{21}}{\sigma_{2}|V|^{2}}\left(\int_{V} \xi \otimes \xi d \xi\right) \cdot \nabla_{x} c=-a_{21} \nabla_{x} c .
$$

Moreover, we use (2.27) to obtain

$$
d_{c}=\frac{1}{\sigma_{1}|V|}\left(\int_{V} \xi \otimes \xi d \xi\right)=\frac{r^{2}}{d \sigma_{1}} I, \quad d_{s}=\frac{1}{\sigma_{2}|V|}\left(\int_{V} \xi \otimes \xi d \xi\right)=\frac{r^{2}}{d \sigma_{1}} I .
$$

Now, we define $G_{i, 1}$, for $i=1,2$ by

$$
\left\{\begin{array}{c}
G_{11}\left(M_{1} c, M_{2} s, \boldsymbol{u}, \xi\right)=\frac{d \sigma_{1}}{r^{2}|V|}\left(M_{1} c \boldsymbol{u}-M_{1}\left(2 a_{11} c+\tilde{d}_{c}(c)\right)-a_{12} M_{2} s\right) \xi \cdot \nabla_{x} c \\
G_{21}\left(M_{1} c, M_{2} s, \boldsymbol{u}, \xi\right)=\frac{d \sigma_{2}}{r^{2}|V|}\left(M_{2} s \boldsymbol{u}-2 a_{21} M_{1} c-M_{2}\left(2 a_{22} s+\tilde{d}_{s}(s)\right)\right) \xi \cdot \nabla_{x} s
\end{array}\right.
$$


where $\tilde{d}_{c}(c)$ and $\tilde{d}_{s}(s)$ are a nonlinear positive functions. Observe that (2.32) satisfies condition (2.9). Next, using relations (2.29) yield

$$
\begin{gathered}
\Gamma_{1}(c, s, \boldsymbol{u})=\frac{d}{r^{2}|V|^{2}}\left(\int_{V} \xi \otimes \xi d \xi\right) \cdot\left(c \boldsymbol{u}-\left(2 a_{11} c+a_{12} s+\tilde{d}_{c}(c)\right) \nabla_{x} c\right)=c \boldsymbol{u}-\left(2 a_{11} c+a_{12} s+\tilde{d}_{c}(c)\right) \nabla_{x} c, \quad(2.33) \\
\Gamma_{2}(c, s, \boldsymbol{u})=\frac{d}{r^{2}|V|^{2}}\left(\int_{V} \xi \otimes \xi d \xi\right) \cdot\left(s \boldsymbol{u}-\left(2 a_{21} c+2 a_{22} s+\tilde{d}_{s}(s)\right) \nabla_{x} s\right)=s \boldsymbol{u}-\left(2 a_{21} c+2 a_{22} s+\tilde{d}_{s}(s)\right) \nabla_{x} s .
\end{gathered}
$$

Now collecting the previous results with $\operatorname{div}_{x} \boldsymbol{u}=0$ and (2.26), we arrive to the macroscopic cross-diffusion-Brinkman model of the order $O(\varepsilon)$

$$
\left\{\begin{array}{l}
c_{t}+\boldsymbol{u} \cdot \nabla c-\operatorname{div}_{x}\left(\left(D_{c}(c)+2 a_{11} c+a_{12} s\right) \nabla_{x} c+a_{12} c \nabla_{x} s\right)=H_{1}(c, s)+O(\varepsilon), \\
s_{t}+\boldsymbol{u} \cdot \nabla s-\operatorname{div}_{x}\left(\left(D_{s}(s)+2 a_{21} c+2 a_{22} s\right) \nabla_{x} s+a_{21} s \nabla_{x} c\right)=H_{2}(c, s)+O(\varepsilon), \\
\mathbb{K}^{-1} \boldsymbol{u}+\sqrt{\mu} \operatorname{curl} \boldsymbol{\omega}+\nabla p=Q(c, s) \boldsymbol{g}+\mathbf{F}, \quad \boldsymbol{\omega}-\sqrt{\mu} \operatorname{curl} \boldsymbol{u}=\mathbf{0}, \quad \operatorname{div}_{x} \boldsymbol{u}=0,
\end{array}\right.
$$

where $D_{c}(c)=\frac{r^{2}}{d \sigma_{1}}+\tilde{d}_{c}(c)$ and $D_{s}(s)=\frac{r^{2}}{d \sigma_{2}}+\tilde{d}_{s}(s)$. In order to derive cross-diffusion-Brinkman with the explicit form of $H_{c}, H_{s}$, we define the interactions operators $G_{12}$ and $G_{22}$ by

$$
\left\{\begin{array}{c}
G_{12}\left(M_{1} c, M_{2} s, \xi\right)=\frac{H_{1}(c, s)}{|V|}, \\
G_{22}\left(M_{1} c, M_{2} s, \xi\right)=\frac{H_{2}(c, s)}{|V|} .
\end{array}\right.
$$

Finally, we use (2.30) to deduce $H_{1}=H_{c}$ and $H_{2}=H_{s}$. Note that we can choose different explicit forms depending on the field of interest (for example, Lotka-Voltera reaction terms as in [6]).

\section{Mathematical ANALYSis of THE CROSS-DIFFUSION-BRINKMAN SYSTEM}

Before stating our result concerning the weak solutions, we collect some preliminary material, including relevant notations and conditions imposed on the data of our problem. Let $\Omega$ be a bounded, open subsets of $\mathbb{R}^{3}$ with a smooth boundary $\partial \Omega ; \eta$ is the unit outward normal to $\Omega$ on $\partial \Omega$. Next, $|\Omega|$ is the Lebesgue measure of $\Omega$. We denote by $H^{1}(\Omega)$ the Sobolev space of functions $u: \Omega \rightarrow \mathbb{R}$ for which $u \in \mathrm{L}^{2}(\Omega)$ and $\nabla u \in \mathrm{L}^{2}\left(\Omega ; \mathbb{R}^{3}\right)$. For $1 \leq p \leq+\infty,\|\cdot\|_{\mathrm{L}^{p}(\Omega)}$ is the usual norm in $L^{p}(\Omega)$; then

$$
\begin{gathered}
\mathrm{L}_{+}^{p}(\Omega)=\left\{u: \Omega \longrightarrow \mathbb{R}_{+} \text {measurable and } \int_{\Omega}|u(x)|^{p} d x<+\infty\right\}, \\
\mathrm{L}_{+}^{\infty}(\Omega)=\left\{u: \Omega \longrightarrow \mathbb{R}_{+} \text {measurable and } \sup _{x \in \Omega}|u(x)|<+\infty\right\} .
\end{gathered}
$$

If $X$ is a Banach space, $a<b$ and $1 \leq p \leq+\infty, \mathrm{L}^{p}(a, b ; X)$ denotes the space of all measurable functions $u:(a, b) \longrightarrow X$ such that $\|u(\cdot)\|_{X}$ belongs to $\mathrm{L}^{p}(a, b)$.

Next $T$ is a positive number and

$$
\Omega_{t}:=\Omega \times(0, t) \text { and } \Sigma_{t}:=\partial \Omega \times(0, t),
$$

for $0<t \leq T$.

We suppose that the permeability tensor $\mathbb{K} \in[C(\bar{\Omega})]^{3 \times 3}$ is symmetric and uniformly positive definite. So it is its inverse, i.e., there exists $C>0$ such that

$$
\boldsymbol{v}^{\mathrm{t}} \mathbb{K}^{-1}(\boldsymbol{x}) \boldsymbol{v} \geq C|\boldsymbol{v}|^{2} \quad \forall \boldsymbol{v} \in \mathbb{R}^{3}, \forall \boldsymbol{x} \in \Omega .
$$

In addition, the diffusivities are assumed always positive, coercive, and continuous: for $i \in\{s, c\}$,

$$
D_{i}:[0,1] \mapsto \mathbb{R}^{+} \text {is continuous, } \quad 0<D^{\min } \leq D_{i}(m) \leq D^{\max }<\infty, \quad m \in \mathbb{R} .
$$

Regarding the function $Q$, we assume it is a continuous function and there exists constant $C_{Q}>0$ such that

$$
|Q(c, s)| \leq C_{Q}(1+|c|+|s|) \text { for all } c, s \in \mathbb{R}
$$


Initial data are assumed nonnegative and in $\mathrm{L}^{2}$

$$
c_{0}, s_{0} \geq 0, \quad c_{0}, s_{0} \in \mathrm{L}^{2}(\Omega) .
$$

In the proof of the existence of the weak solution, we will use the following assumption

$$
8 a_{11} a_{21} \geq a_{21}^{2} \text { and } 8 a_{22} a_{12} \geq a_{21} .
$$

Let us also consider the kernel of the bilinear form $\int_{\Omega} q \operatorname{div} \boldsymbol{u} \mathrm{d} \boldsymbol{x}$, that is

$$
\mathrm{X}:=\left\{\boldsymbol{v} \in \mathbf{H}_{0}(\operatorname{div} ; \Omega): \int_{\Omega} q \operatorname{div} \boldsymbol{v} \mathrm{d} \boldsymbol{x}=0, \forall q \in \mathrm{L}_{0}^{2}(\Omega)\right\}=\left\{\boldsymbol{v} \in \mathbf{H}_{0}(\operatorname{div} ; \Omega): \operatorname{div} \boldsymbol{v}=0 \text { a.e. in } \Omega\right\} .
$$

Moreover, we endow the space $\mathbf{H}(\mathbf{c u r l} ; \Omega)$ with the following $\mu$-dependent norm:

$$
\|\boldsymbol{z}\|_{\mathbf{H}(\mathbf{c u r l} ; \Omega)}^{2}:=\|\boldsymbol{z}\|_{0, \Omega}^{2}+\mu\|\operatorname{curl} \boldsymbol{z}\|_{0, \Omega}^{2},
$$

and recall the following inf-sup condition ( $c f$. [27]): there exists $\beta>0$ only depending on $\Omega$, such that

$$
\sup _{\substack{\boldsymbol{v} \in \mathbf{H}(\operatorname{div} ; \Omega) \\ \boldsymbol{v} \neq 0}} \frac{\left|\int_{\Omega} q \operatorname{div} \boldsymbol{v} \mathrm{d} \boldsymbol{x}\right|}{\|\boldsymbol{v}\|_{\mathbf{H}(\operatorname{div} ; \Omega)}} \geq \beta\|q\|_{0, \Omega} \quad \forall q \in \mathrm{L}_{0}^{2}(\Omega) .
$$

Now we define what we mean by weak solutions of the system (1.3). We also supply our main existence result.

Definition 3.1. We say that $(c, s, \boldsymbol{u}, \boldsymbol{\omega}, p)$ is a weak solution to problem (1.3), if $c$ and $s$ are nonnegative,

$$
c, s \in \mathrm{L}^{\infty}\left(0, T ; \mathrm{L}^{2}(\Omega)\right) \cap \mathrm{L}^{2}\left(0, T ; \mathrm{H}^{1}(\Omega)\right)
$$

$$
\boldsymbol{u} \in \mathbf{L}^{2}(0, T ; \mathbf{H}(\operatorname{div} ; \Omega)), \quad \boldsymbol{\omega} \in \mathbf{L}^{2}(0, T ; \mathbf{H}(\operatorname{curl} ; \Omega)), p \in \mathrm{L}^{2}\left(0, T ; \mathrm{L}_{0}^{2}(\Omega)\right)
$$

and

$$
\begin{aligned}
\int_{0}^{T}\left\langle\partial_{t} c, \varphi_{c}\right\rangle d t+\iint_{\Omega_{T}}\left[\left(D_{c}(c)+2 a_{11} c+a_{12} s\right) \nabla c+a_{12} c \nabla s-c \boldsymbol{u}\right] \cdot \nabla \varphi_{c} d \boldsymbol{x} d t & =\iint_{\Omega_{T}} H_{c}(c, s) \varphi_{c} d \boldsymbol{x} d t \\
\int_{0}^{T}\left\langle\partial_{t} s, \varphi_{s}\right\rangle d t+\iint_{\Omega_{T}}\left[\left(D_{s}(s)+2 a_{21} c+2 a_{22} s\right) \nabla s+a_{21} s \nabla-s \boldsymbol{u}\right] \cdot \nabla \varphi_{s} d \boldsymbol{x} d t & =\iint_{\Omega_{T}} H_{s}(c, s) \varphi_{s} d \boldsymbol{x} d t \\
\iint_{\Omega_{T}} \mathbb{K}^{-1} \boldsymbol{u} \cdot \boldsymbol{v} d x d t+\sqrt{\mu} \iint_{\Omega_{T}} \operatorname{curl} \boldsymbol{\omega} \cdot \boldsymbol{v} d \boldsymbol{x} d t-\iint_{\Omega_{T}} p \operatorname{div} \boldsymbol{v} d \boldsymbol{x} d t & =\iint_{\Omega_{T}}(Q(c, s) \boldsymbol{g}+\boldsymbol{F}) \cdot \boldsymbol{v} d \boldsymbol{x} d t \\
\sqrt{\mu} \iint_{\Omega_{T}} \operatorname{curl} \boldsymbol{z} \cdot \boldsymbol{u} d \boldsymbol{x} d t-\iint_{\Omega_{T}} \boldsymbol{\omega} \cdot \boldsymbol{z} d \boldsymbol{x} d t & =0 \\
-\iint_{\Omega_{T}} q \operatorname{div} \boldsymbol{u} d \boldsymbol{x} d t & =0
\end{aligned}
$$

for all $\varphi_{c}, \varphi_{s} \in \mathrm{L}^{2}\left(0, T ; W^{1, \infty}(\Omega)\right), \boldsymbol{v} \in \mathbf{L}^{2}\left(0, T ; \mathbf{H}_{0}(\operatorname{div} ; \Omega)\right), \boldsymbol{z} \in \mathbf{L}^{2}\left(0, T ; \mathbf{H}_{0}(\mathbf{c u r l} ; \Omega)\right)$, $q \in \mathrm{L}^{2}\left(0, T ; \mathrm{L}_{0}^{2}(\Omega)\right)$. Here, $\langle\cdot, \cdot\rangle$ denotes the duality pairing between $W^{1, \infty}(\Omega)$ and $\left(W^{1, \infty}(\Omega)\right)^{*}$.

Theorem 3.1. Assume conditions (3.1), (3.2), (3.5) and (3.6) hold. If $c_{0} \in \mathrm{L}_{+}^{2}(\Omega), s_{0} \in \mathrm{L}_{+}^{2}(\Omega)$, then the problem (1.3) possesses a weak solution.

To prove Theorem 3.1, we first prove existence of solutions to the approximate problem (3.7) below by applying the Schauder fixed-point theorem (in an appropriate functional setting). Then, having proved existence for the aproximate system, the final goal is to send the regularization parameter $\varepsilon$ to zero to fabricate weak solutions of the original systems (1.3). Convergence is achieved by means of a priori estimates and compactness arguments.

Remark 3.1. Note that a major difficulty for our model (1.3) is the strong coupling in the highest derivatives. Therefore, standard parabolic theory is not directly applicable to our system due to the cross-diffusion-Brinkman terms. We point out that this model is strongly nonlinear and so no maximum principle applies. Moreover, we have not been able to prove uniqueness of weak solutions because of the presence of nonlinear lower-order terms (cross-diffusion terms). 
3.1. Existence of solutions for the approximate problems. This subsection is devoted to proving existence of solutions to the approximate problem (3.7) below of system (1.3). The existence proof is based on the Shauder fixed-point theorem, a priori estimates, and the compactness method. The approximation systems read for $(t, \boldsymbol{x}) \in(0, T] \times \Omega$ :

$$
\left\{\begin{array}{l}
c_{t}+\boldsymbol{u} \cdot \nabla c-\operatorname{div}\left[\left(D_{c}(c)+2 a_{11} f_{\varepsilon}^{+}(c)+a_{12} f_{\varepsilon}^{+}(s)\right) \nabla c+a_{12} f_{\varepsilon}^{+}(c) \nabla s\right]=H_{c, \varepsilon}\left(c^{+}, s^{+}\right), \\
s_{t}+\boldsymbol{u} \cdot \nabla s-\operatorname{div}\left[\left(D_{s}(s)+2 a_{21} f_{\varepsilon}^{+}(c)+2 a_{22} f_{\varepsilon}^{+}(s)\right) \nabla s+a_{21} f_{\varepsilon}^{+}(s) \nabla c\right]=H_{s, \varepsilon}\left(c^{+}, s^{+}\right), \\
\mathbb{K}^{-1} \boldsymbol{u}+\sqrt{\mu} \operatorname{curl} \boldsymbol{\omega}+\nabla p=Q(s, c) \boldsymbol{g}+\mathbf{F}, \quad \boldsymbol{\omega}-\sqrt{\mu} \operatorname{curl} \boldsymbol{u}=\mathbf{0}, \quad \operatorname{div}_{x} \boldsymbol{u}=0,
\end{array}\right.
$$

subject to the boundary conditions and initial data given by (1.4). Herein, $\varepsilon>0$ is a small number,

$$
\left\{\begin{array}{l}
H_{c, \varepsilon}(a)=\frac{H_{c}}{1+\varepsilon\left|H_{c}\right|} \text { and } H_{s, \varepsilon}(a)=\frac{H_{s}}{1+\varepsilon\left|H_{s}\right|}, \\
f_{\varepsilon}(a)=\frac{a}{1+\varepsilon|a|} \text { and } b^{+}=\max (0, b) \text { for any } a, b \in \mathbb{R} .
\end{array}\right.
$$

Note that under condition (3.5), the matrix

$$
M(c, s)=\left(\begin{array}{cc}
\left(2 a_{11} f^{+}(c)+a_{12} f^{+}(s)\right) & \frac{1}{2}\left(a_{12} f^{+}(c)+a_{21} f^{+}(s)\right. \\
\frac{1}{2}\left(a_{12} f^{+}(c)+a_{21} f(s)\right) & \left(a_{21} f^{+}(c)+2 a_{22} f^{+}(c)\right)
\end{array}\right)
$$

is uniformly nonnegative (see [16] for more details). We shall frequently use this to prove the existence (and nonnegativity) of weak solutions.

3.2. Existence result to the fixed problem. In this subsection, we omit the dependence of the solutions on the parameter $\varepsilon$. We prove, for each fixed $\varepsilon>0$, the existence of solutions to the fixed problem (3.7), by applying the Schauder fixed-point theorem. Since we use Schauder fixed-point theorem, we need to introduce the following closed subset of the Banach space $\mathrm{L}^{2}\left(\Omega_{T}, \mathbb{R}^{n}\right)$ :

$$
\mathcal{A}=\left\{U=(c, s) \in \mathrm{L}^{2}\left(\Omega_{T}, \mathbb{R}^{2}\right):\|U\|_{\mathrm{L}^{\infty}\left(0, T ; \mathrm{L}^{2}\left(\Omega, \mathbb{R}^{2}\right)\right) \cap \mathrm{L}^{2}\left(0, T ; \mathrm{H}^{1}\left(\Omega, \mathbb{R}^{2}\right)\right)} \leq C_{\mathcal{A}}\right\},
$$

where $C_{\mathcal{A}}>0$ is a constant that will be defined below. With $(\bar{c}, \bar{s}) \in \mathcal{A}$ fixed, let $(c, s, \boldsymbol{u}, \boldsymbol{\omega}, p)$ be the unique solution of the system in $\Omega_{T}$

$$
\left\{\begin{array}{l}
c_{t}+\boldsymbol{u} \cdot \nabla c-\operatorname{div}\left[\left(D_{c}(\bar{c})+2 a_{11} f_{\varepsilon}^{+}(\bar{c})+a_{12} f_{\varepsilon}^{+}(\bar{s})\right) \nabla c+a_{12} f_{\varepsilon}^{+}(\bar{c}) \nabla s\right]=H_{c, \varepsilon}\left(\bar{c}^{+}, \bar{s}^{+}\right), \\
s_{t}+\boldsymbol{u} \cdot \nabla s-\operatorname{div}\left[\left(D_{s}(\bar{s})+2 a_{21} f_{\varepsilon}^{+}(\bar{c})+2 a_{22} f_{\varepsilon}^{+}(\bar{s})\right) \nabla s+a_{21} f_{\varepsilon}^{+}(\bar{s}) \nabla c\right]=H_{s, \varepsilon}\left(\bar{c}^{+}, \bar{s}^{+}\right), \\
\mathbb{K}^{-1} \boldsymbol{u}+\sqrt{\mu} \operatorname{curl} \boldsymbol{\omega}+\nabla p=Q(\bar{c}, \bar{s}) \boldsymbol{g}+\mathbf{F}, \quad \boldsymbol{\omega}-\sqrt{\mu} \operatorname{curl} \boldsymbol{u}=\mathbf{0}, \quad \operatorname{div}{ }_{x} \boldsymbol{u}=0 .
\end{array}\right.
$$

3.3. The fixed-point method. Now, we introduce a map $\mathrm{L}: \mathcal{A} \rightarrow \mathcal{A}$ such that $\mathrm{L}(\bar{c}, \bar{s})=(c, s)$, where $(c, s)$ solve (3.9). By using the Schauder fixed-point theorem, we prove that the map L have a fixed point for (3.9).

We start with the following result where the proof can be found in [27] Theorem 1.3:

Theorem 3.2. Let $\left(\mathcal{X},\langle\cdot, \cdot\rangle_{\mathcal{X}}\right)$ be a Hilbert space. Let $\mathcal{A}: \mathcal{X} \times \mathcal{X} \rightarrow \mathbb{R}$ be a bounded symmetric bilinear form, and let $\mathcal{G}: \mathcal{X} \rightarrow \mathbb{R}$ be a bounded functional. Assume that there exists $\bar{\beta}>0$ such that

$$
\sup _{\substack{y \in \mathcal{X} \\ y \neq 0}} \frac{\mathcal{A}(x, y)}{\|y\|_{\mathcal{X}}} \geq \bar{\beta}\|x\|_{\mathcal{X}} \quad \forall x \in \mathcal{X} .
$$

Then, there exists a unique $x \in \mathcal{X}$, such that

$$
\mathcal{A}(x, y)=\mathcal{G}(y) \quad \forall y \in \mathcal{X} .
$$

Moreover, there exists $C>0$, independent of the solution, such that

$$
\|x\|_{\mathcal{X}} \leq C\|\mathcal{G}\|_{\mathcal{X}^{\prime}} .
$$


Observe that from Brinkman equation in (3.9) we solve the following problem: Find $(\boldsymbol{u}, \boldsymbol{\omega}) \in$ $\mathrm{X} \times \mathbf{H}(\mathbf{c u r l} ; \Omega)$ such that

$$
\begin{gathered}
\int_{\Omega} \mathbb{K}^{-1} \boldsymbol{u} \cdot \boldsymbol{v} \mathrm{d} \boldsymbol{x}+\sqrt{\mu} \int_{\Omega} \operatorname{curl} \boldsymbol{\omega} \cdot \boldsymbol{v} \mathrm{d} \boldsymbol{x}=\int_{\Omega}(Q(\bar{c}, \bar{s}) \boldsymbol{g}+\mathbf{F}) \cdot \boldsymbol{v} \mathrm{d} \boldsymbol{x}, \quad \forall \boldsymbol{v} \in \mathrm{X}, \\
\sqrt{\mu} \int_{\Omega} \operatorname{curl} \boldsymbol{z} \cdot \boldsymbol{u} \mathrm{d} \boldsymbol{x}-\int_{\Omega} \boldsymbol{\omega} \cdot \boldsymbol{z} \mathrm{d} \boldsymbol{x}=0, \quad \forall \boldsymbol{z} \in \mathbf{H}_{0}(\operatorname{curl} ; \Omega) .
\end{gathered}
$$

Next we exploit Theorem 3.2 and we work exactly as in the proofs of [5, Theorem 2.2 and Corollary 2.1] to get the following lemma for a fixed $(\bar{c}, \bar{s}) \in \mathcal{A}$ and for any $t>0$.

Lemma 3.1. Assume that $(\bar{c}, \bar{s}) \in \mathcal{A}$. Then, the variational problem

$$
\begin{aligned}
\int_{\Omega} \mathbb{K}^{-1} \boldsymbol{u} \cdot \boldsymbol{v} \mathrm{d} \boldsymbol{x}+\sqrt{\mu} \int_{\Omega} \operatorname{curl} \boldsymbol{\omega} \cdot \boldsymbol{v} \mathrm{d} \boldsymbol{x}-\int_{\Omega} p \operatorname{div} \boldsymbol{v} \mathrm{d} \boldsymbol{x}=\int_{\Omega}(Q(\bar{c}, \bar{s}) \boldsymbol{g}+\boldsymbol{F}) \cdot \boldsymbol{v} \mathrm{d} \boldsymbol{x}, \\
\sqrt{\mu} \int_{\Omega} \operatorname{curl} \boldsymbol{z} \cdot \boldsymbol{u} \mathrm{d} \boldsymbol{x}-\int_{\Omega} \boldsymbol{\omega} \cdot \boldsymbol{z} \mathrm{d} \boldsymbol{x}=0, \\
-\int_{\Omega} q \operatorname{div} \boldsymbol{u} \mathrm{d} \boldsymbol{x}=0,
\end{aligned}
$$

admits a unique solution $(\boldsymbol{u}, \boldsymbol{\omega}, p) \in \mathbf{H}(\operatorname{div} ; \Omega) \times \mathbf{H}(\mathbf{c u r l} ; \Omega) \times \mathrm{L}_{0}^{2}(\Omega)$. Moreover, there exists $C>0$ independent of $\mu$ such that

$\|\boldsymbol{u}\|_{\mathbf{H}(\operatorname{div} ; \Omega)}+\|\boldsymbol{\omega}\|_{\mathbf{H}(\mathbf{c u r l} ; \Omega)}+\|p\|_{0, \Omega} \leq C\left(\left(1+\|\bar{c}\|_{0, \Omega}+\|\bar{s}\|_{0, \Omega}\right)\|\boldsymbol{g}\|_{\infty, \Omega}+\|\boldsymbol{F}\|_{0, \Omega}+\left\|u_{\partial}\right\|_{-1 / 2, \partial \Omega}+\left\|\boldsymbol{\omega}_{\partial}\right\|_{-1 / 2, \partial \Omega}\right)$.

Now, let us show that $\mathrm{L}$ is a continuous mapping. For this, letting $\left(\bar{c}_{\ell}, \bar{s}_{\ell}\right)_{\ell}$ be sequence in $\mathcal{A}$. Next, we let $(\bar{c}, \bar{s}) \in \mathcal{A}$ be such that $\left(\bar{c}_{\ell}, \bar{s}_{\ell}\right)_{\ell} \rightarrow(\bar{c}, \bar{s})$ in $\mathrm{L}^{2}\left(\Omega_{T}, \mathbb{R}^{2}\right)$ as $\ell \rightarrow \infty$. Define $\left(c_{\ell}, s_{\ell}\right)=\mathrm{L}\left(\bar{c}_{\ell}, \bar{s}_{\ell}\right)$. The goal is to show that $\left(c_{\ell}, s_{\ell}\right)$ converges to $\mathrm{L}(\bar{c}, \bar{s})$ in $\mathrm{L}^{2}\left(\Omega_{T}, \mathbb{R}^{3}\right)$. Next, we need the following lemma:

Lemma 3.2. The solution $\left(c_{\ell}, s_{\ell}\right)$ to system (3.9) satisfies:

(i) The sequence $\left(c_{\ell}, s_{\ell}\right)_{\ell}$ is bounded in $\mathrm{L}^{2}\left(0, T ; \mathrm{H}^{1}\left(\Omega, \mathbb{R}^{2}\right)\right) \cap \mathrm{L}^{\infty}\left(0, T ; \mathrm{L}^{2}\left(\Omega, \mathbb{R}^{2}\right)\right)$.

(ii) The sequence $\left(c_{\ell}, s_{\ell}\right)_{\ell}$ is relatively compact in $\mathrm{L}^{2}\left(\Omega_{T}, \mathbb{R}^{3}\right)$.

Proof. ( $i$ ) We multiply the first and the second in (3.9) by $c_{\ell}$ and $s_{\ell}$ respectively, integrate over $\Omega$ and using the uniform nonnegativity of $M\left(\bar{c}_{\ell}, \bar{s}_{\ell}\right)$, yields

$$
\begin{aligned}
\frac{d}{d t} \int_{\Omega}\left(\left|c_{\ell}\right|^{2}+\left|s_{\ell}\right|^{2}\right) d \boldsymbol{x}+D^{\min } \int_{\Omega}\left(\left|\nabla c_{\ell}\right|^{2}+\left|\nabla s_{\ell}\right|^{2}\right) d \boldsymbol{x} & \leq \int_{\Omega} H_{c, \varepsilon}\left(\bar{c}_{\ell}^{+}, \bar{s}_{\ell}^{+}\right) c_{\ell} d \boldsymbol{x}+\int_{\Omega} H_{s, \varepsilon}\left(\bar{c}_{\ell}^{+}, \bar{s}_{\ell}^{+}\right) s_{\ell} d \boldsymbol{x} \\
& \leq C \int_{\Omega}\left(\left|c_{\ell}\right|^{2}+\left|s_{\ell}\right|^{2}\right) d \boldsymbol{x}
\end{aligned}
$$

for some constant $C>0$. Where we have used assumptions (3.2) and (3.5), and

$$
\int_{\Omega} c_{\ell} \boldsymbol{u} \cdot \nabla c_{\ell} \mathrm{d} \boldsymbol{x}+\int_{\Omega} s_{\ell} \boldsymbol{u} \cdot \nabla s_{\ell} \mathrm{d} \boldsymbol{x}=\frac{1}{2} \int_{\Omega} \boldsymbol{u} \cdot \nabla\left(c_{\ell}\right)^{2} \mathrm{~d} \boldsymbol{x}+\frac{1}{2} \int_{\Omega} \boldsymbol{u} \cdot \nabla\left(s_{\ell}\right)^{2} \mathrm{~d} \boldsymbol{x}=0 .
$$

In view of Gronwall's inequality it follows from (3.12) that,

$$
\sup _{t \in(0, T)} \int_{\Omega}\left(\left|c_{\ell}\right|^{2}+\left|s_{\ell}\right|^{2}\right) d \boldsymbol{x} \leq \exp (C T)\left\|c_{0}+s_{0}\right\|_{L^{2}(\Omega)},
$$

which proves the first part of (i).

From (3.12) and (3.13) we may also conclude that,

$$
\iint_{\Omega_{T}}\left(\left|\nabla c_{\ell}\right|^{2}+\left|\nabla s_{\ell}\right|^{2}\right) d \boldsymbol{x} d t \leq \frac{T \exp (C T)}{D^{\min }}\left\|c_{0}+s_{0}\right\|_{L^{2}(\Omega)},
$$

yielding (i). 
(ii) Finally multiplying the first, the second and the third equation (3.9) by $\varphi_{c}, \varphi_{s} \in \mathrm{L}^{2}\left(0, T ; \mathrm{H}^{1}(\Omega)\right)$, respectively and using the boundedness of $f_{\varepsilon}^{+}, H_{c, \varepsilon}, H_{s, \varepsilon}$, and (3.14) there exists a constant $C(\varepsilon)>0$ such that

$$
\left|\int_{0}^{T}\left\langle\partial_{t} c_{\ell}, \varphi_{c}\right\rangle d t\right|+\left|\int_{0}^{T}\left\langle\partial_{t} s_{\ell}, \varphi_{s}\right\rangle d t\right| \leq C(\varepsilon)\left(\left\|\varphi_{c}\right\|_{\mathrm{L}^{2}\left(0, T ; \mathrm{H}^{1}(\Omega)\right)}+\left\|\varphi_{c}\right\|_{\mathrm{L}^{2}\left(0, T ; \mathrm{H}^{1}(\Omega)\right)}\right),
$$

so we get (ii). Then, (ii) is a consequence of (i) and the uniform boundedness (3.15) of $\left(c_{\ell}, s_{\ell}\right)_{\ell}$ in $\mathrm{L}^{2}\left(0, T ;\left(\mathrm{H}^{1}\left(\Omega, \mathbb{R}^{2}\right)\right)^{\prime}\right)$

Remark 3.2. Note that it is easy to deduce from Lemma 3.2 that the constant $C_{\mathcal{A}}>0$ (consult (3.8)) is defined as follows:

$$
C_{\mathcal{A}}=\frac{\left(D^{\mathrm{min}}+T\right) \exp (C T)}{D^{\min }}\left\|c_{0}+s_{0}\right\|_{\mathrm{L}^{2}(\Omega)}
$$

for some constant $C>0$.

From Lemma 3.2, there exist functions $\left(c_{\ell}, s_{\ell}\right) \in \mathrm{L}^{2}\left(0, T ; \mathrm{H}^{1}\left(\Omega, \mathbb{R}^{2}\right)\right)$ such that, up to extracting subsequences if necessary,

$$
\left(c_{\ell}, s_{\ell}\right) \rightarrow(c, s) \text { in }\left(\mathrm{L}^{2}\left(\Omega_{T}\right)\right)^{2} \text { strongly, }
$$

and from this the continuity of $\mathrm{L}$ on $\mathcal{A}$ follows.

We observe that, from Lemma $3.2, \mathrm{~L}(\mathcal{A})$ is bounded in the set

$$
\mathcal{E}=\left\{u \in \mathrm{L}^{2}\left(0, T ; \mathrm{H}^{1}\left(\Omega, \mathbb{R}^{2}\right)\right): \partial_{t} u \in \mathrm{L}^{2}\left(0, T ;\left(\mathrm{H}^{1}\left(\Omega, \mathbb{R}^{2}\right)\right)^{\prime}\right)\right\}
$$

By the results of [39], $\mathcal{E} \hookrightarrow \mathrm{L}^{2}\left(\Omega_{T}, \mathbb{R}^{2}\right)$ is compact, thus $\mathrm{L}$ is compact. Now, by the Schauder fixed point theorem, the operator $\mathrm{L}$ has a fixed point $\left(c_{\varepsilon}, s_{\varepsilon}\right)$ such that $\mathrm{L}\left(c_{\varepsilon}, s_{\varepsilon}\right)=\left(c_{\varepsilon}, s_{\varepsilon}\right)$. Then there exists a solution $\left(c_{\varepsilon}, s_{\varepsilon}, \boldsymbol{u}_{\varepsilon}, \boldsymbol{\omega}_{\varepsilon}, p_{\varepsilon}\right)$ of

$$
\begin{aligned}
& \left.\int_{0}^{T}\left\langle\partial_{t} c_{\varepsilon}, \varphi_{c}\right\rangle d t+\iint_{\Omega_{T}}\left[\left(D_{c}\left(c_{\varepsilon}\right)+2 a_{11} f_{\varepsilon}^{+}\left(c_{\varepsilon}\right)+a_{12} f_{\varepsilon}^{+} s_{\varepsilon}\right)\right) \nabla c_{\varepsilon}+a_{12} f_{\varepsilon}^{+}\left(c_{\varepsilon}\right) \nabla s-c_{\varepsilon} \boldsymbol{u}_{\varepsilon}\right] \cdot \nabla m_{\varepsilon}^{c} d \boldsymbol{x} d t \\
& =\iint_{\Omega_{T}} H_{c, \varepsilon}\left(c_{\varepsilon}^{+}, s_{\varepsilon}^{+}\right) \varphi_{c} d \boldsymbol{x} d t \\
& \int_{0}^{T}\left\langle\partial_{t} s_{\varepsilon}, \varphi_{s}\right\rangle d t+\iint_{\Omega_{T}}\left[\left(D_{s}\left(s_{\varepsilon}\right)+2 a_{21} f_{\varepsilon}^{+}\left(c_{\varepsilon}\right)+2 a_{22} f_{\varepsilon}^{+}\left(s_{\varepsilon}\right)\right) \nabla s_{\varepsilon}+a_{21} f_{\varepsilon}^{+}\left(s_{\varepsilon}\right) \nabla c_{\varepsilon}-c_{\varepsilon} \boldsymbol{u}_{\varepsilon}\right] \cdot \nabla \varphi_{s} d \boldsymbol{x} d t \\
& =\iint_{\Omega_{T}} H_{s, \varepsilon}\left(c_{\varepsilon}^{+}, s_{\varepsilon}^{+}\right) \varphi_{s} d \boldsymbol{x} d t \\
& \iint_{\Omega_{T}} \mathbb{K}^{-1} \boldsymbol{u}_{\varepsilon} \cdot \boldsymbol{v} d x d t+\sqrt{\mu} \iint_{\Omega_{T}} \operatorname{curl} \boldsymbol{\omega}_{\varepsilon} \cdot \boldsymbol{v} d \boldsymbol{x} d t-\iint_{\Omega_{T}} p_{\varepsilon} \operatorname{div} \boldsymbol{v} d \boldsymbol{x} d t=\iint_{\Omega_{T}}\left(Q\left(c_{\varepsilon}, s_{\varepsilon}\right) \boldsymbol{g}+\mathbf{F}\right) \cdot \boldsymbol{v} d \boldsymbol{x} d t \\
& \sqrt{\mu} \iint_{\Omega_{T}} \operatorname{curl} \boldsymbol{z} \cdot \boldsymbol{u}_{\varepsilon} d \boldsymbol{x} d t-\iint_{\Omega_{T}} \boldsymbol{\omega}_{\varepsilon} \cdot \boldsymbol{z} d \boldsymbol{x} d t=0, \\
& -\iint_{\Omega_{T}} q \operatorname{div} \boldsymbol{u}_{\varepsilon} d \boldsymbol{x} d t=0
\end{aligned}
$$

for all $\varphi_{c}, \varphi_{s} \in \mathrm{L}^{2}\left(0, T ; \mathrm{H}^{1}(\Omega)\right), \boldsymbol{v} \in \mathbf{L}^{2}\left(0, T ; \mathbf{H}_{0}(\operatorname{div} ; \Omega)\right), \boldsymbol{z} \in \mathbf{L}^{2}\left(0, T ; \mathbf{H}_{0}(\mathbf{c u r l} ; \Omega)\right), q \in \mathrm{L}^{2}\left(0, T ; \mathrm{L}_{0}^{2}(\Omega)\right)$.

3.4. Existence of weak solutions. Note that from Subsection 3.3, we know there exist sequences $\left(c_{\varepsilon}, s_{\varepsilon}, \boldsymbol{u}_{\varepsilon}, \boldsymbol{\omega}_{\varepsilon}, p_{\varepsilon}\right)_{\varepsilon>0}$ solution to (3.7). We have now the following series of a priori estimates.

Lemma 3.3. Assume conditions (3.1), (3.2) and (3.5) hold. If $c_{0}, s_{0} \in L_{+}^{2}(\Omega)$ then the solution $\left(c_{\varepsilon}, s_{\varepsilon}, \boldsymbol{u}_{\varepsilon}, \boldsymbol{\omega}_{\varepsilon}, p_{\varepsilon}\right)$ is nonnegative. Moreover, there exist constants $c_{1}, \ldots, c_{5}>0$ not depending on $\varepsilon$ such that

$$
\begin{gathered}
\left\|\left(c_{\varepsilon}, s_{\varepsilon}\right)\right\|_{\mathrm{L}^{\infty}\left(0, T ; \mathrm{L}^{2}\left(\Omega, \mathbb{R}^{2}\right)\right)} \leq c_{1}, \\
\left\|H_{c, \varepsilon}\left(c_{\varepsilon}, s_{\varepsilon}\right)\right\|_{\mathrm{L}^{1}\left(\Omega_{T}\right)}+\left\|H_{s, \varepsilon}\left(c_{\varepsilon}, s_{\varepsilon}\right)\right\|_{\mathrm{L}^{1}\left(\Omega_{T}\right)} \leq c_{2}, \\
\left\|\nabla c_{\varepsilon}\right\|_{\mathrm{L}^{2}\left(\Omega_{T}\right)}+\left\|\nabla s_{\varepsilon}\right\|_{\mathrm{L}^{2}\left(\Omega_{T}\right)} \leq c_{3},
\end{gathered}
$$




$$
\begin{gathered}
\left\|\boldsymbol{u}_{\varepsilon}\right\|_{\mathbf{H}(\operatorname{div} ; \Omega)}+\left\|\boldsymbol{\omega}_{\varepsilon}\right\|_{\mathbf{H}(\mathbf{c u r l} ; \Omega)}+\left\|p_{\varepsilon}\right\|_{0, \Omega} \leq c_{4}, \\
\left\|\partial_{t} c_{\varepsilon}\right\|_{\mathrm{L}^{2}\left(0, T,\left(W^{1, \infty}\left(\Omega_{1}\right)\right)^{*}\right)}+\left\|\partial_{t} s_{\varepsilon}\right\|_{\mathrm{L}^{2}\left(0, T,\left(W^{1, \infty}\left(\Omega_{1}\right)\right)^{*}\right)} \leq c_{5} .
\end{gathered}
$$

Proof.

In the weak formulation (3.17) we take $\varphi_{c}=-c_{\varepsilon}^{-}, \varphi_{s}=-s_{\varepsilon}^{-}$, and we integrate over $\Omega$ instead $\Omega_{T}$, we get from (3.2) and (3.5)

$$
\frac{1}{2} \frac{d}{d t} \int_{\Omega}\left(\left|c_{\varepsilon}^{-}\right|^{2}+\left|s_{\varepsilon}^{-}\right|^{2}\right) \mathrm{d} \boldsymbol{x} \leq 0
$$

Herein, we used

$$
-\int_{\Omega} c_{\varepsilon}^{-} \boldsymbol{u}_{\varepsilon} \cdot \nabla c_{\varepsilon} \mathrm{d} \boldsymbol{x}-\int_{\Omega} s_{\ell} \boldsymbol{u}_{\varepsilon} \cdot \nabla s_{\varepsilon} \mathrm{d} \boldsymbol{x}=\frac{1}{2} \int_{\Omega} \boldsymbol{u}_{\varepsilon} \cdot \nabla\left(c_{\varepsilon}^{-}\right)^{2} \mathrm{~d} \boldsymbol{x}+\frac{1}{2} \int_{\Omega} \boldsymbol{u}_{\varepsilon} \cdot \nabla\left(s_{\varepsilon}^{-}\right)^{2} \mathrm{~d} \boldsymbol{x}=0 .
$$

This yields the nonnegativity of $\left(c_{\varepsilon}, s_{\varepsilon}\right)$.

By the (weak) lower semicontinuity properties of norms, the estimates (3.13) and (3.14) hold with $\left(c_{\ell}, c_{\ell}\right)$ replaced by $\left(c_{\varepsilon}, s_{\varepsilon}\right)$. Moreover, the constants $c_{1}, c_{3}$ are independent of $\varepsilon$ (consult the proof of Lemma 3.2). Observe that for $j=c, s$

$$
\left|H_{j, \varepsilon}\left(c_{\varepsilon}, s_{\varepsilon}\right)\right| \leq C\left(\left|c_{\varepsilon}\right|^{2}+\left|s_{\varepsilon}\right|^{2}\right),
$$

for some constant $C>0$. Using this, (3.19) is a consequence of (3.18).

Next, we use (3.18) and Lemma 3.1 to deduce (3.21). Finally using the weak formulation (3.17), we deduce from (3.18) and (3.20): for all

$\phi_{1} \in \mathrm{L}^{2}\left(0, T ; W^{1, \infty}\left(\Omega_{1}\right)\right)$

$$
\begin{aligned}
& \mid \int_{0}^{T}\left\langle\partial_{t} c_{\varepsilon}, \varphi_{c}\right\rangle \mathrm{d} t \\
& \leq D^{\max }\left\|\nabla c_{\varepsilon}\right\|_{\mathrm{L}^{2}\left(\Omega_{T}\right)}\left\|\nabla \varphi_{c}\right\|_{\mathrm{L}^{2}\left(\Omega_{T}\right)}+\left\|c_{\varepsilon}\right\|_{\mathrm{L}^{\infty}\left(0, T ; \mathrm{L}^{2}(\Omega)\right)}\left\|\boldsymbol{u}_{\ell}\right\|_{\mathrm{L}^{2}\left(\Omega_{T}\right)}\left\|\nabla \varphi_{c}\right\|_{\mathrm{L}^{2}\left(0, T ; W^{1, \infty}(\Omega)\right)} \\
&+C \|\left(\left\|c_{\varepsilon}\right\|_{\mathrm{L}^{\infty}\left(0, T ; \mathrm{L}^{2}(\Omega)\right)}+\left\|s_{\varepsilon}\right\|_{\mathrm{L}^{\infty}\left(0, T ; \mathrm{L}^{2}(\Omega)\right)}\right) \\
& \times\left(\boldsymbol{u}_{\ell}+\left\|\nabla s_{\varepsilon}\right\|_{\mathrm{L}^{2}\left(\Omega_{T}\right)}\right)\left\|\nabla \varphi_{c}\right\|_{\mathrm{L}^{2}\left(0, T ; \mathrm{L}^{\infty}(\Omega)\right)} \\
&+C^{\prime}\left(1+\left\|c_{\varepsilon}\right\|_{\mathrm{L}^{\infty}\left(0, T ; \mathrm{L}^{2}(\Omega)\right)}+\left\|s_{\varepsilon}\right\|_{\mathrm{L}^{\infty}\left(0, T ; \mathrm{L}^{2}(\Omega)\right)}\right) \\
& \times\left(1+\left\|c_{\varepsilon}\right\|_{\mathrm{L}^{2}\left(\Omega_{T}\right)}+\left\|s_{\varepsilon}\right\|_{\mathrm{L}^{2}\left(\Omega_{T}\right)}\right)\left\|\varphi_{c}\right\|_{\mathrm{L}^{2}\left(0, T ; \mathrm{L}^{\infty}(\Omega)\right)} \\
& \leq C^{\prime \prime}\left\|\varphi_{c}\right\|_{\mathrm{L}^{2}\left(0, T ; W^{1, \infty}(\Omega)\right)}
\end{aligned}
$$

for some constant $C, C^{\prime}, C^{\prime \prime}>0$ independent of $\varepsilon$. From this we deduce the bound

$$
\left\|\partial_{t} c_{\varepsilon}\right\|_{\mathrm{L}^{2}\left(0, T ;\left(W^{1, \infty}(\Omega)\right)^{*}\right)} \leq C^{\prime \prime}
$$

Reasoning along the same lines for $c_{\varepsilon}$ yields (3.25) for $s_{\varepsilon}$.

In view of Lemma 3.3 and Aubin's lemma, we can assume there exist limit functions $(c, s)$ such that as $\varepsilon \rightarrow 0$ the following convergences hold (modulo extraction of subsequences, which we do not bother to relabel):

$$
\left\{\begin{array}{l}
\left(c_{\varepsilon}, s_{\varepsilon}\right) \rightarrow(c, s) \text { a.e. in } \Omega_{T}, \text { and strongly in } \mathrm{L}^{2}\left(\Omega_{T}, \mathbb{R}^{2}\right), \text { weakly in } \mathrm{L}^{2}\left(0, T ; \mathrm{H}^{1}\left(\Omega, \mathbb{R}^{2}\right)\right) \\
H_{j, \varepsilon}\left(c_{\varepsilon}, s_{\varepsilon}\right) \rightarrow H_{j, c}(c, s) \text { a.e. in } \Omega_{T} \text { and strongly in } \mathrm{L}^{1}\left(\Omega_{T}\right) \\
\boldsymbol{u}_{\varepsilon} \rightarrow \boldsymbol{u} \text { weakly in } \mathbf{L}^{2}\left(0, T ; \mathbf{H}_{0}(\operatorname{div} ; \Omega)\right) \\
\boldsymbol{\omega}_{\varepsilon} \rightarrow \boldsymbol{\omega} \text { weakly in } \mathbf{L}^{2}\left(0, T ; \mathbf{H}_{0}(\mathbf{c u r l} ; \Omega)\right) \\
p_{\varepsilon} \rightarrow p \text { weakly in } \mathrm{L}^{2}\left(0, T ; \mathrm{L}_{0}^{2}(\Omega)\right)
\end{array}\right.
$$


for $j=c, s$. Additionally, $\left(\partial_{t} c_{\varepsilon}, \partial_{t} s_{\varepsilon},\right) \rightarrow\left(\partial_{t} c, \partial_{t} s\right)$ weakly in $\mathrm{L}^{2}\left(0, T ;\left(W^{1, \infty}\left(\Omega, \mathbb{R}^{2}\right)\right)^{*}\right)$ as $\varepsilon \rightarrow 0$. An application of Young and Hölder inequalities we get

$$
\begin{aligned}
\left\|f_{\varepsilon}\left(c_{\varepsilon}\right)-c\right\|_{L^{2}\left(\Omega_{T}\right)} & \leq \sqrt{2}\left\|c_{\varepsilon}-c\right\|_{L^{2}\left(\Omega_{T}\right)}+\sqrt{2}\left\|\frac{\varepsilon c_{\varepsilon} c}{1+\varepsilon c_{\varepsilon}}\right\|_{L^{2}\left(\Omega_{T}\right)} \\
& \leq \sqrt{2}\left\|c_{\varepsilon}-c\right\|_{L^{2}\left(\Omega_{T}\right)}+\sqrt{2}\left\|\frac{\varepsilon \varepsilon_{\varepsilon} c}{\left(1+\varepsilon c_{\varepsilon}\right)^{2 / 3}\left(\varepsilon c_{\varepsilon}\right)^{1-2 / 3}}\right\|_{L^{2}\left(\Omega_{T}\right)} \\
& \leq \sqrt{2}\left\|c_{\varepsilon}-c\right\|_{L^{2}\left(\Omega_{T}\right)}+\sqrt{2} \varepsilon^{2 / 3}\left\|c_{\varepsilon}^{2 / 3} c\right\|_{L^{2}\left(\Omega_{T}\right)} \\
& \leq \sqrt{2}\left\|c_{\varepsilon}-c\right\|_{L^{2}\left(\Omega_{T}\right)}+\sqrt{2} \varepsilon^{2 / 3}\left\|c_{\varepsilon}\right\|_{L^{\infty}\left(0, T ; L^{2}(\Omega)\right)}^{2 / \pi}\left(\|c\|_{L^{2}\left(0, T ; L^{6}(\Omega)\right)} .\right.
\end{aligned}
$$

Thanks to the Sobolev embedding $\left(H^{1}(\Omega) \subset L^{6}(\Omega)\right)$ we deduce from $(3.27)$

$$
f_{\varepsilon}\left(c_{\varepsilon}\right) \rightarrow c \text { a.e. in } \Omega_{T} \text { and strongly in } \mathrm{L}^{2}\left(\Omega_{T}\right) .
$$

In the same way we get

$$
f_{\varepsilon}\left(s_{\varepsilon}\right) \rightarrow s \text { a.e. in } \Omega_{T} \text { and strongly in } L^{2}\left(\Omega_{T}\right) .
$$

Finally, by passing to the limit $\varepsilon \rightarrow 0$ in the weak formulation (3.17), with $\varphi_{c}, \varphi_{s} \in \mathrm{L}^{2}\left(0, T ; W^{1, \infty}(\Omega)\right)$, $\boldsymbol{v} \in \mathbf{L}^{2}\left(0, T ; \mathbf{H}_{0}(\operatorname{div} ; \Omega)\right), \boldsymbol{z} \in \mathbf{L}^{2}\left(0, T ; \mathbf{H}_{0}(\mathbf{c u r l} ; \Omega)\right)$ and $q \in \mathrm{L}^{2}\left(0, T ; \mathrm{L}_{0}^{2}(\Omega)\right)$, we obtain in this way that the limit $(c, s, \boldsymbol{u}, \boldsymbol{\omega}, p)$ is a solution of system problem (1.3) in the sense of Definition 3.1.

\section{NUMERICAL ANALYSIS OF MICRO-MACRO CROSS-DIFFUSION-BRINKMAN SYSTEM}

In this section we develop an asymptotic preserving scheme (AP). We propose a numerical scheme uniformly stable along the transition from kinetic to macroscopic regimes. Inspiring by the continuous derivation, we use the time semi-implicit discretization for the micro-macro formulation (2.17) which is equivalent to the kinetic-fluid model (2.1). After the full discretization of (2.17), we show various numerical tests to validate the proposed approach.

4.1. A time semi-implicit discretization. First, we present a time discretization to our coupled system (2.17). We denote by $\Delta t$ a fixed time step and by $t_{k}:=k \Delta t$ a discrete time with $k \in \mathbb{N}$. The approximations of $c(t, x), s(t, x), g_{1}(t, x, \xi), g_{2}(t, x, \xi), \boldsymbol{u}(t, x), \boldsymbol{\omega}(t, x)$ and $p(t, x)$ at the time step $t_{k}$ are denoted by $c^{k}:=c\left(t_{k}, x\right), s^{k}:=s\left(t_{k}, x\right), g_{i}^{k}:=g_{i}\left(t_{k}, x, \xi\right), \boldsymbol{u}^{k}:=\boldsymbol{u}\left(t_{k}, x\right), \boldsymbol{\omega}^{k}:=\boldsymbol{\omega}\left(t_{k}, x\right)$ and $p^{k}:=p\left(t_{k}, x\right)$ for $i=1,2$. The semi-implicit scheme for microscopic equations in system (2.17) reads

$$
\left\{\begin{array}{c}
\frac{g_{1}^{k+1}-g_{1}^{k}}{\Delta t}+\frac{1}{\varepsilon^{2}} \xi M_{1} \cdot \nabla c^{k}+\frac{1}{\varepsilon}\left(I-P_{M_{1}}\right)\left(\xi \cdot \nabla g_{1}^{k}\right)=\frac{1}{\varepsilon^{2}} \mathcal{L}_{1}\left(g_{1}^{k+1}\right)+ \\
\frac{1}{\varepsilon^{2}} \mathcal{T}_{1}^{2}\left[M_{2}(\xi) s^{k}\right]\left(M_{1}(\xi) c^{k}\right)+\frac{1}{\varepsilon} \mathcal{T}_{1}^{2}\left[M_{2}(\xi) s^{k}\right]\left(g_{1}^{k}\right)+ \\
\frac{1}{\varepsilon^{2}} G_{11}\left(M_{1}(\xi) c^{k}, M_{2}(\xi) s^{k}, \boldsymbol{u}^{k}, \xi\right)+\frac{1}{\varepsilon}\left(I-P_{M_{1}}\right) G_{12}\left(M_{1}(\xi) c^{k}, M_{2}(\xi) s^{k}, \xi\right) \\
\frac{g_{2}^{k+1}-g_{2}^{k}}{\Delta t}+\frac{1}{\varepsilon^{2}} \xi M_{2} \cdot \nabla s^{k}+\frac{1}{\varepsilon}\left(I-P_{M_{2}}\right)\left(\xi \cdot \nabla_{x} g_{2}^{k}\right)=\frac{1}{\varepsilon^{2}} \mathcal{L}_{2}\left(g_{2}^{k+1}\right)+ \\
\frac{1}{\varepsilon^{2}} \mathcal{T}_{2}^{2}\left[M_{1}(\xi) c^{k}\right]\left(M_{2}(\xi) s^{k}\right)+\frac{1}{\varepsilon} \mathcal{T}_{2}^{2}\left[M_{1}(\xi) c^{k}\right]\left(g_{2}^{k}\right)+ \\
\frac{1}{\varepsilon^{2}} G_{21}\left(M_{1}(\xi) c^{k}, M_{2}(\xi) s^{k}, \boldsymbol{u}^{k}, \xi\right)+\frac{1}{\varepsilon}\left(I-P_{M_{2}}\right) G_{22}\left(M_{1}(\xi) c^{k}, M_{2}(\xi) s^{k}, \xi\right)
\end{array}\right.
$$


In the hydrodynamic equations of system (2.17), we take $g_{1}$ and $g_{2}$ at the time $t_{k+1}$. The result is

$$
\left\{\begin{array}{l}
\frac{c^{k+1}-c^{k}}{\Delta t}+\left\langle\xi \cdot \nabla_{x} g_{1}^{k+1}\right\rangle=\left\langle G_{12}\left(M_{1}(\xi) c^{k}, M_{2}(\xi) s^{k}, \xi\right)\right\rangle, \\
\frac{s^{k+1}-s^{k}}{\Delta t}+\left\langle\xi \cdot \nabla g_{2}^{k+1}\right\rangle=\left\langle G_{22}\left(M_{1}(\xi) c^{k+1}, M_{2}(\xi) s^{k}, \xi\right)\right\rangle . \\
\mathbb{K}^{-1} \boldsymbol{u}^{k+1}+\sqrt{\mu} \operatorname{curl} \boldsymbol{\omega}^{k+1}+\nabla p^{k+1}=Q\left(c^{k+1}, s^{k+1}\right) \boldsymbol{g}+\mathbf{F}, \\
\boldsymbol{\omega}^{k+1}-\sqrt{\mu} \operatorname{curl} \boldsymbol{u}^{k+1}=\mathbf{0}, \quad \operatorname{div}_{x} \boldsymbol{u}^{k+1}=0 .
\end{array}\right.
$$

Proposition 4.1. The numerical scheme given by (4.1)-(4.2) is consistent with equations (2.25) when $\varepsilon$ goes to 0 .

Proof. The asymptotic behavior of scheme (4.1)-(4.2) is obtained similarly as the continuous case. Since the operator $-\mathcal{L}_{i}$ is self adjoint and positive, the operator $\left(I-\frac{\Delta t}{\epsilon^{2}} \mathcal{L}_{i}\right)$ is also invertible for all $\Delta t \geq 0$ for $i=1,2$. Hence (4.1) gives

$$
\begin{gathered}
g_{1}^{k+1}=\left(I-\frac{\Delta t}{\epsilon^{2}} \mathcal{L}_{1}\right)^{-1}\left[g_{1}^{k}+\frac{\Delta t}{\varepsilon^{2}}\left(\mathcal{T}_{1}^{2}\left[M_{2} s^{k}\right]\left(M_{1} c^{k}+\varepsilon g_{1}^{k}\right)+G_{11}\left(M_{1} c^{k}, M_{2} s^{k}, \boldsymbol{u}^{k}, \xi\right)\right.\right. \\
\left.\left.-\xi M_{1} \cdot \nabla c^{k}\right)+\frac{\Delta t}{\varepsilon}\left(I-P_{M_{1}}\right)\left(G_{12}\left(M_{1} c^{k}, M_{2} s^{k}, \xi\right)-\xi \cdot \nabla g_{1}^{k}\right)\right]
\end{gathered}
$$

and

$$
\begin{gathered}
g_{2}^{k+1}=\left(I-\frac{\Delta t}{\epsilon^{2}} \mathcal{L}_{2}\right)^{-1}\left[g_{2}^{k}+\frac{\Delta t}{\varepsilon^{2}}\left(\mathcal{T}_{2}^{2}\left[M_{1} c^{k}\right]\left(M_{2}(\xi) s^{k}+\varepsilon g_{2}^{k}\right)+G_{21}\left(M_{1} c^{k}, M_{2} s^{k}, \boldsymbol{u}^{k}, \xi\right)\right.\right. \\
\left.\left.-\xi M_{2} \cdot \nabla s^{k}\right)+\frac{\Delta t}{\varepsilon}\left(I-P_{M_{2}}\right)\left(G_{22}\left(M_{1} c^{k}, M_{2} s^{k}, \xi\right)-\xi \cdot \nabla g_{2}^{k}\right)\right] .
\end{gathered}
$$

It is easy to see that $\left(I-\frac{\Delta t}{\varepsilon^{2}} \mathcal{L}_{i}\right)^{-1}=\frac{\Delta t}{\varepsilon^{2}} \mathcal{L}_{i}^{-1}+O\left(\varepsilon^{3}\right)$ for $i=1,2$. Using this, we get

$$
g_{1}^{k+1}=\mathcal{L}_{1}^{-1}\left(\xi M_{1} \cdot \nabla c^{k}-\mathcal{T}_{1}^{2}\left[M_{2} s^{k}\right]\left(M_{1} c^{k}\right)-G_{11}\left(M_{1} c^{k}, M_{2} s^{k}, \boldsymbol{u}^{k}, \xi\right)\right)+O(\varepsilon),
$$

and

$$
\left.g_{2}^{k+1}=\mathcal{L}_{2}^{-1}\left(\xi M_{2} \cdot \nabla s^{k}-\mathcal{T}_{2}^{2}\left[M_{1} c^{k}\right]\left(M_{2} s^{k}\right)\right)-G_{21}\left(M_{1} c^{k}, M_{2} s^{k}, \boldsymbol{u}^{k}, \xi\right)\right)+O(\varepsilon) .
$$

Inserting $g_{1}^{k+1}$ and $g_{2}^{k+1}$ into (4.2), we obtain

$$
\begin{gathered}
\frac{c^{k+1}-c^{k}}{\Delta t}+\left\langle\xi \cdot \nabla \mathcal{L}_{1}^{-1}\left[\xi M_{1} \cdot \nabla c^{k}-\mathcal{T}_{1}^{2}\left[M_{2} s^{k}\right]\left(M_{1} c^{k}\right)-G_{11}\left(M_{1} c^{k}, M_{2} s^{k}, \boldsymbol{u}^{k}, \xi\right)\right]\right\rangle \\
=\left\langle G_{12}\left(M_{1} c^{k}, M_{2} s^{k}, \xi\right)\right\rangle+O(\varepsilon)
\end{gathered}
$$

and

$$
\begin{gathered}
\frac{s^{k+1}-s^{k}}{\Delta t}+\left\langle\xi \cdot \nabla \mathcal{L}_{2}^{-1}\left[\xi M_{2} \cdot \nabla s^{k}-\mathcal{T}_{2}^{2}\left[M_{1} c^{k}\right]\left(M_{2} s^{k}\right)-G_{21}\left(M_{1} c^{k}, M_{2} s^{k}, \boldsymbol{u}^{k}, \xi\right)\right]\right\rangle \\
=\left\langle G_{22}\left(M_{1} c^{k+1}, M_{2} s^{k}, \xi\right)\right\rangle+O(\varepsilon),
\end{gathered}
$$

which is consistent with system $(2.25)$ when $\varepsilon$ goes to 0 . 
4.2. Full discretization. In this subsection, we present our method in one-dimensional case into the domain $[-a, a]$ for fixed $a \in \mathbb{R}$. Let $\mathbb{T}=\left\{K_{j}, \quad j=1, \ldots, N_{x}\right\}$ be an admissible mesh in the meaning of Definition 5.5 page 125 in Ref. [26]. The control volume is given by $\left.K_{j}=\right] x_{j-\frac{1}{2}}, x_{j+\frac{1}{2}}$ [ with $x_{j}=\frac{1}{2}\left(x_{j-\frac{1}{2}}+x_{j+\frac{1}{2}}\right)$ and its length is denoted by $h_{j}=x_{j+\frac{1}{2}}-x_{j-\frac{1}{2}}$ for $j=1, \ldots, N_{x}$. For the velocity space, we consider $\xi_{\ell}=\xi_{\min }+\ell \Delta \xi$, for $\ell=0, \ldots, N_{\xi}-1$ where $\Delta \xi=\frac{1}{N_{\xi}}\left(\xi_{\max }-\xi_{\min }\right)$ with $\xi_{\max }=-\xi_{\min }$. We shall assume that $\boldsymbol{g}=\mathbf{F}=0$. Thus the fluid velocity is a given function depending only on time, namely $u(t)=\partial_{x} p$. The methodology is as follow: the macroscopic equations in (4.2) are computed in the control volume $K_{j}$ while the microscopic equations in (4.1) should be computed in the interface of $K_{j}$. Precisely, the macroscopic densities are as follow

$$
\left.c\left(t_{k}, x\right)\right|_{K_{j}} \approx c_{j}^{k},\left.\quad s\left(t_{k}, x\right)\right|_{K_{j}} \approx s_{j}^{k}
$$

and the microscopic ones are given by

$$
\left.g_{1}\left(t_{k}, x_{j+\frac{1}{2}}, \xi_{\ell}\right)\right|_{\partial K_{j}} \approx g_{1, j+\frac{1}{2}, \ell}^{k},\left.\quad g_{2}\left(t_{k}, x_{j+\frac{1}{2}}, \xi_{\ell}\right)\right|_{\partial K_{j}} \approx g_{2, j+\frac{1}{2}, \ell}^{k}
$$

for $j=1, \ldots, N_{x}$ and $\ell=1, \ldots, N_{\xi}$.

Now, we integrate over the control volume $K_{j}$ the macroscopic equations in (4.2), we approximate the time derivatives by differential quotients and using an upwind choice for $g_{1}$ and $g_{2}$ to arrive

$$
\left\{\begin{array}{l}
\frac{c_{j}^{k+1}-c_{j}^{k}}{\Delta t}+\left\langle\xi_{\ell} \frac{g_{1, j+\frac{1}{2}, \ell}^{k+1}-g_{1, j-\frac{1}{2}, \ell}^{k+1}}{h_{j}}\right\rangle=\left\langle G_{12}\left(M_{1, \ell} c_{j}^{k}, M_{2, \ell} s_{j}^{k}, \xi_{\ell}\right)\right\rangle, \\
\frac{s_{j}^{k+1}-s_{j}^{k}}{\Delta t}+\left\langle\xi_{\ell} \frac{g_{2, j+\frac{1}{2}, \ell}^{k+1}-g_{2, j-\frac{1}{2}, \ell}^{k+1}}{h_{j}}\right\rangle=\left\langle G_{22}\left(M_{1, \ell} c_{j}^{k+1}, M_{2, \ell} s_{j}^{k}, \xi_{\ell}\right)\right\rangle, \\
\frac{p_{j+1}^{k}-p_{j}^{k}}{h_{j}}=u^{k} .
\end{array}\right.
$$

For the microscopic equations in (4.1), we compute the unknowns functions $g_{1}$ and $g_{2}$ in the interface of $K_{j}$ (or integrating over the control volume $I_{j+\frac{1}{2}}$ ):

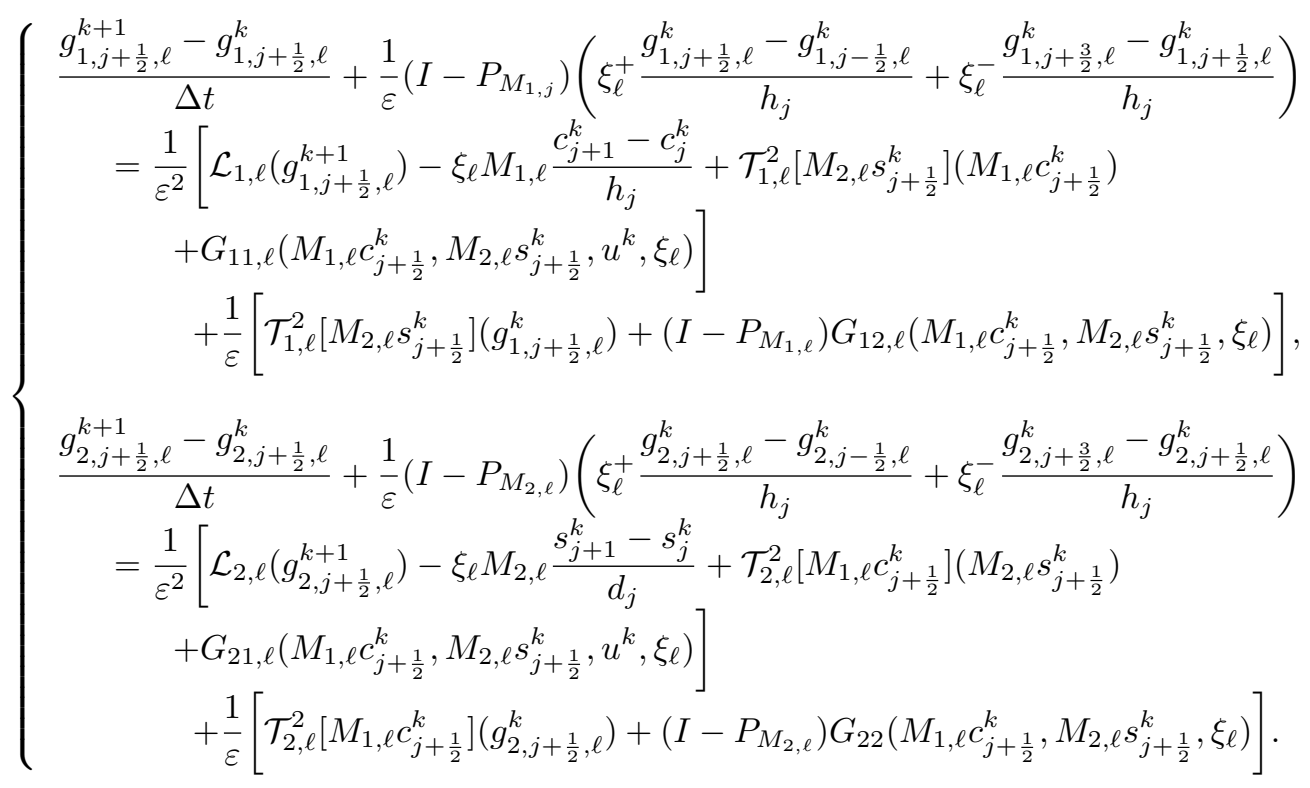

In the following proposition, we show that our scheme proposed in the micro-macro formulation (4.8)-(4.7) is uniformly stable along the transition from kinetic to macroscopic regimes. 
Proposition 4.2. The time and space approximations (4.8)-(4.7) of the micro-macro formulation in the limit ( $\varepsilon$ goes to zero), satisfy the following discretization:

$$
\begin{gathered}
\frac{c_{j}^{k+1}-c_{j}^{k}}{\Delta t}+\frac{1}{h_{j}}\left\langle\xi_{\ell}\left[\mathcal{L}_{1, \ell}^{-1}\left(\xi_{\ell} M_{1, \ell} \frac{c_{j+1}^{k}-c_{j}^{k}}{h_{j}}\right)-\mathcal{L}_{1, \ell}^{-1}\left(\xi_{\ell} M_{1, \ell} \frac{c_{j}^{k}-c_{j-1}^{k}}{h_{j}}\right)\right]\right\rangle \\
+\frac{1}{h_{j}}\left\langle\xi_{\ell}\left[\mathcal{L}_{1, \ell}^{-1}\left(\mathcal{T}_{1}^{2}\left[M_{2, \ell} s_{j+\frac{1}{2}}^{k}\right]\left(M_{1, \ell} c_{j+\frac{1}{2}}^{k}\right)\right)-\mathcal{L}_{1, \ell}^{-1}\left(\mathcal{T}_{1, \ell}^{2}\left[M_{2, \ell} s_{j-\frac{1}{2}}^{k}\right]\left(M_{1, \ell} c_{j-\frac{1}{2}}^{k}\right)\right)\right]\right\rangle \\
\left.\left.+\frac{1}{h_{j}}\left\langle\xi_{\ell}\left[\mathcal{L}_{1, \ell}^{-1}\left(G_{11, \ell}\left(M_{1, \ell} c_{j+\frac{1}{2}}^{k}, M_{2, \ell} s_{j+\frac{1}{2}}^{k}, u^{k}, \xi_{\ell}\right)\right)\right)-\mathcal{L}_{1, \ell}^{-1}\left(G_{11, \ell}\left(M_{1, \ell} c_{j-\frac{1}{2}}^{k}, M_{2, \ell} s_{j-\frac{1}{2}}^{k}, u^{k}, \xi_{\ell}\right)\right)\right)\right]\right\rangle \\
=\left\langle G_{12, \ell}\left(M_{1, \ell} c_{j}^{k}, M_{2, \ell} s_{j}^{k}, \xi_{\ell}\right)\right\rangle+O(\varepsilon),
\end{gathered}
$$

and

$$
\begin{array}{r}
\frac{s_{j}^{k+1}-s_{j}^{k}}{\Delta t}+\frac{1}{h_{j}}\left\langle\xi_{\ell}\left[\mathcal{L}_{2, \ell}^{-1}\left(\xi_{\ell} M_{2, \ell} \frac{s_{j+1}^{k}-s_{j}^{k}}{h_{j}}\right)-\mathcal{L}_{2, \ell}^{-1}\left(\xi_{\ell} M_{2, \ell} \frac{s_{j}^{k}-s_{j-1}^{k}}{h_{j}}\right)\right]\right\rangle \\
+\frac{1}{h_{j}}\left\langle\xi_{\ell}\left[\mathcal{L}_{2, \ell}^{-1}\left(\mathcal{T}_{2, \ell}^{2}\left[M_{1, \ell} c_{j+\frac{1}{2}}^{k}\right]\left(M_{2, \ell} s_{j+\frac{1}{2}}^{k}\right)\right)-\mathcal{L}_{2, \ell}^{-1}\left(\mathcal{T}_{2, \ell}^{2}\left[M_{1, \ell} c_{j-\frac{1}{2}}^{k}\right]\left(M_{2, \ell} s_{j-\frac{1}{2}}^{k}\right)\right)\right]\right\rangle \\
\left.\left.+\frac{1}{h_{j}}\left\langle\xi_{\ell}\left[\mathcal{L}_{2, \ell}^{-1}\left(G_{21, \ell}\left(M_{1, \ell} c_{j+\frac{1}{2}}^{k}, M_{2, \ell} s_{j+\frac{1}{2}}^{k}, u^{k}, \xi_{\ell}\right)\right)\right)-\mathcal{L}_{2, \ell}^{-1}\left(G_{21, \ell}\left(M_{1, \ell} c_{j-\frac{1}{2}}^{k}, M_{2, \ell} s_{j-\frac{1}{2}}^{k}, u^{k}, \xi_{\ell}\right)\right)\right)\right]\right\rangle \\
=\left\langle G_{22, \ell}\left(M_{2, \ell} c_{j}^{k+1}, M_{2, \ell} s_{j}^{k}, \xi_{\ell}\right)\right\rangle+O(\varepsilon),
\end{array}
$$

which is consistent with system (2.25).

4.3. Boundary conditions. For the numerical solution of the kinetic equation (2.1), the following inflow boundary conditions are usually prescribe for the distribution functions $f_{1}$ and $f_{2}$ :

$$
f_{i}\left(t, x_{\min }, v\right)=f_{i, l}(v), \quad v>0 \quad f_{i}\left(t, x_{\max }, v\right)=f_{i, r}(v), \quad v<0, \text { for } i=1,2 .
$$

We shall denote $w_{1}=c$ and $w_{2}=s$. The inflow boundary conditions can be rewritten in the micro-macro formulation by

$$
\begin{gathered}
w_{i}\left(t, x_{0}\right) M_{i}+\frac{\varepsilon}{2}\left(g_{i}\left(t, x_{\frac{1}{2}}, v\right)+g_{i}\left(t, x_{-\frac{1}{2}}, v\right)\right)=f_{i, l}(v), \quad v<0, \\
w_{i}\left(t, x_{N_{x}}\right) M_{i}+\frac{\varepsilon}{2}\left(g_{i}\left(t, x_{N_{x}+\frac{1}{2}}, v\right)+g_{i}\left(t, x_{N_{x}-\frac{1}{2}}, v\right)\right)=f_{i, r}(v), \quad v>0,
\end{gathered}
$$

for $i=1,2$. For the other velocities, we consider the following artificial Neumann boundary conditions:

$$
\begin{gathered}
g_{i}\left(t, x_{\frac{1}{2}}, v_{\ell}\right)=g_{i}\left(t, x_{-\frac{1}{2}}, v_{\ell}\right), \quad v_{\ell}<0, \\
g_{i}\left(t, x_{N_{x}+\frac{1}{2}}, v_{\ell}\right)=g_{i}\left(t, x_{N_{x}-\frac{1}{2}}, v_{\ell}\right), \quad v_{\ell}>0,
\end{gathered}
$$

for $i=1,2$. Furthermore, the ghost points can be computed for $i=1,2$ as follows:

$$
\begin{aligned}
& g_{i, \frac{1}{2}, \ell}^{k+1}=\left\{\begin{array}{l}
\frac{2}{\varepsilon}\left(f_{1, l}\left(v_{\ell}\right)-w_{i, 0}^{k+1} M_{i}\right)-g_{i, \frac{1}{2}, \ell}^{k+1}, \quad v_{\ell}>0, \\
g_{i, \frac{1}{2}, \ell}^{k+1}, \quad v_{\ell}<0 ;
\end{array}\right. \\
& g_{i, N_{x}+\frac{1}{2}, \ell}^{k+1}\left\{\begin{array}{l}
\frac{2}{\varepsilon}\left(f_{i, r}\left(v_{\ell}\right)-w_{i, N_{x}}^{k+1} M_{i}\right)-g_{i, N_{x}-\frac{1}{2}}^{k+1}, \quad v_{\ell}<0, \\
g_{i, N_{x}-\frac{1}{2}, \ell}^{k+1}, \quad v_{\ell}>0 .
\end{array}\right.
\end{aligned}
$$

It then follows from (4.7) that for $i=1,2$ :

$$
\left\{\begin{aligned}
\left(1+\frac{2 \Delta t}{\varepsilon h_{0}}<v_{\ell}^{+} M_{i, \ell}>\right) w_{i, 0}^{k+1}= & w_{i, 0}^{k}-\frac{\Delta t}{h_{0}}\left\langle\left(v_{\ell}+v_{\ell}^{+}-v_{\ell}^{-}\right) g_{i, \frac{1}{2}, \ell}^{k+1}-\frac{2 v_{\ell}^{+}}{\varepsilon} f_{i, l}\left(v_{\ell}\right)\right\rangle \\
& +\Delta t\left\langle G_{i 2, \ell}\left(M_{1, \ell} c_{0}^{k}, M_{2, \ell} s_{0}^{k}\right)\right\rangle, \\
\left(1-\frac{2 \Delta t}{\varepsilon h_{N_{x}}}<v_{\ell}^{-} M_{i, \ell}>\right) w_{i, N_{x}}^{k+1} & =w_{i, N_{x}}^{k}-\frac{\Delta t}{h_{N_{x}}}\left\langle\frac{2 v_{\ell}^{-}}{\varepsilon} f_{i, r}\left(v_{\ell}\right)-\left(v_{\ell}-v_{\ell}^{+}+v_{\ell}^{-}\right) g_{i, N_{x}-\frac{1}{2}, \ell}^{k+1}\right\rangle \\
& +\Delta t\left\langle G_{i 2, \ell}\left(M_{1, \ell} c_{N_{x}}^{k}, M_{2, \ell} s_{N_{x}}^{k}\right)\right\rangle .
\end{aligned}\right.
$$


4.4. Numerical results. In this subsection, we present some numerical experiments in order to validate our approach. In the following tests, the computational domain in space is $[-1 ; 1]$ while the velocity space is $V=[-1 ; 1]$ with 64 discrete points which can provide good enough for numerical simulations [19]. We adopt a set of parameters, namely the coefficients of intra- and inter-specific competition, used in the book by [40] (adopted also by [6] ): $a_{1}=0.61 /$ year, $a_{2}=0.82 /$ year, $b_{1}=0.4575, b_{2}=0.31, d_{1}=9.5$ and $d_{2}=8.2$. The diffusion coefficients are constants $\left(D_{c}=1\right.$ and $\left.D_{s}=1\right)$. Moreover, we consider $a_{11}=a_{21}=a_{22}=0.5$ and $a_{12}=1$ which satisfy the conditions (3.5). The initial densities corresponds to the $c$-predator species and the $s$-prey species are given by

$$
c_{0}(x)=0.65 \quad \text { and } \quad s_{0}(x)=\exp \left(-30 x^{2}\right) .
$$

The initial cell distribution function is as follow

$$
f_{1}(0 ; x ; \xi)=c_{0}(x) M_{1}(\xi) \quad \text { and } \quad f_{2}(0 ; x ; \xi)=s_{0}(x) M_{2}(\xi),
$$

where $M_{i}(\xi)=\frac{1}{|V|}$ for $i=1,2$.

Next, we consider the macroscopic cross-diffusion-Brinkman model in one dimension. We discretize this model by using finite volume method

$$
\left\{\begin{aligned}
& \frac{c_{j}^{k+1}-c_{j}^{k}}{\Delta t}=D_{c}\left(\frac{c_{j+1}^{k}-c_{j}^{k}}{h_{j} h_{i+\frac{1}{2}}}-\frac{c_{j}^{k}-c_{j-1}^{k}}{h_{i-\frac{1}{2}} h_{j}}\right)-\frac{\left(\partial_{x}^{(m)} c_{j+1}^{k}\right)(c+s)_{j+1}^{k}-\left(\partial_{x}^{(m)} c_{j-1}^{k}\right)(c+s)_{j-1}^{k}}{h_{j}} \\
&-\frac{c_{j+1}^{k}-c_{j}^{k}}{h_{j}} u^{k}+2 \frac{\left(\partial_{x}^{(m)} s_{j+1}^{k}\right) c_{j+1}^{k}-\left(\partial_{x}^{(m)} s_{j-1}^{k}\right) c_{j-1}^{k}}{h_{j}}+H_{1}\left(c_{j}^{k}, s_{j}^{k}\right), \\
& \frac{s_{j}^{k+1}-s_{j}^{k}}{\Delta t}=D_{s}\left(\frac{s_{j+1}^{k}-s_{j}^{k}}{h_{j} h_{i+\frac{1}{2}}}-\frac{s_{j}^{k}-s_{j-1}^{k}}{h_{i-\frac{1}{2}} h_{j}}\right)-\frac{\left(\partial_{x}^{(m)} s_{j+1}^{k}\right)\left(c^{k+1}+s^{k}\right){ }_{j+1}-\left(\partial_{x}^{(m)} s_{j-1}^{k}\right)\left(c^{k+1}+s^{k}\right)_{j-1}}{h_{j}} \\
& \quad-\frac{s_{j+1}^{k}-s_{j}^{k}}{h_{j}} u^{k}+0.5 \frac{\left(\partial_{x}^{(m)} c_{j+1}^{k+1}\right) s_{j+1}^{k}-\left(\partial_{x}^{(m)} c_{j-1}^{k+1}\right) s_{j-1}^{k}}{h_{j}}+H_{2}\left(c_{j}^{k+1}, s_{j}^{k}\right), \\
& u^{k+1}=\frac{p_{j+1}^{k}-p_{j}^{k}}{h_{j}} .
\end{aligned}\right.
$$

where

$$
\partial_{x}^{(m)} z_{j}^{k}=\frac{z_{j+1}^{k}-z_{j}^{k}}{h_{j}} \quad \text { and } \quad \partial_{x}^{(m)} z_{0}^{k}=\partial_{x}^{(m)} z_{N_{x}}^{k}=0,
$$

for $1 \leq j \leq N_{x}-1$. In Figure 1, we present the plots in log scale of the error estimates given by

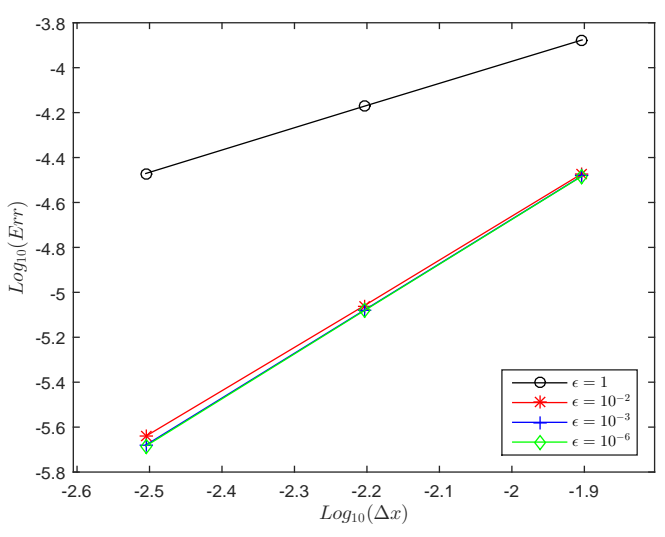

(a)

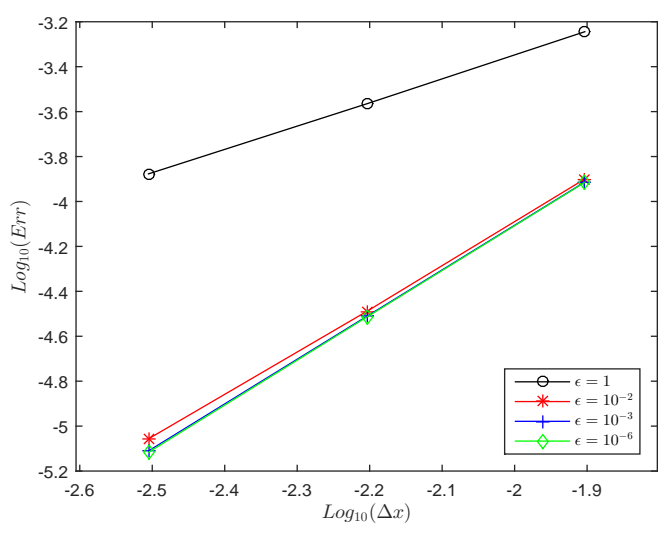

(b)

Figure 1. Convergence order of the method for $\varepsilon \in\left\{1,10^{-2}, 10^{-3}, 10^{-6}\right\}$ at time $t=0.01(M=1)$ for the density $c$ in the left and the density $s$ in the right obtained from micro-macro scheme. 


$$
e_{\Delta x}(h)=\frac{\left|h_{\Delta x}(t)-h_{2 \Delta x}(t)\right|_{1}}{\left|h_{2 \Delta x}(0)\right|_{1}}
$$

to test the convergence of our scheme. This can be considered as an estimation of the relative error in $l^{1}$ norm, where $h_{\Delta x}$ is the numerical solution computed from a uniform grid of size $\Delta x=\frac{x_{\max }-x_{\min }}{N_{x}}$. The computations are performed with $N_{x}=\{80,160,320,640\}, \Delta t=10^{-6}$ at $t=0.01$ for $\varepsilon=\left\{1,10^{-2}, 10^{-3}, 10^{-6}\right\}$.

In Figure 2, we compute our numerical scheme for different values of times $t=0.02,0.03,0.07,0.1$ and for $\varepsilon=10^{-k}$ with $k \in\{0,1,2,3,6\}$ against the macroscopic model in the case: $u=0$ and $c_{0}=0.65, s_{0}=\exp \left(30 x^{2}\right)$. Moreover in Figure 3, we consider $u=1$ and $c_{0}=0.65, s_{0}=$ $\exp \left(30(x+0.5)^{2}\right)$. These figures show that our (AP) scheme is stable in the limit (when $\varepsilon \rightarrow 0$ ). We observe that the profile of the densities $c$ and $s$ given by the two schemes are almost the same when $\varepsilon \rightarrow 0$ and this illustrates the result in Proposition 4.2. Moreover, we observe that the crossdiffusion effect induces the formation of patterns (by using Turing mechanisms ) in the presence of the fluid. Moreover, the distribution of prey and predator is affected by the fluid transport.

In Figures 4, 5 and 6, we illustrate the evolution of the densities $c$ and $s$ using micro-macro scheme for $\varepsilon=10^{-6}$ at final time $T=0.01$ with different values of $u(u=0, u=1$ and $u=-1)$. We note here that the species are diffusing according to the sign of the velocity $u$.

\section{Conclusion And Perspectives}

In this paper, we have proposed a new nonlinear macroscopic model coupled with the augmented Brinkman problem in a viscous flow in porous media. Specifically, the micro-macro decomposition has been applied to the kinetic system coupled with Brinkman problem (2.1) to derive asymptotic preserving numerical scheme. In other parts, we have proved the existence of weak solutions of the derived model (1.3) by using Schauder fixed-point theory. Finally, it has shown that the presented numerical scheme enjoys the asymptotic preserving property in the following meaning: when Knudsen parameter $\varepsilon$ is small, our scheme is asymptotically equivalent to a standard numerical scheme for the derived macroscopic model. In this work, we developed our numerical results in one dimension using finite volume method for both macroscopic model (cross-diffusion-Brinkman) and micro-macro formulation. We believe that our technique can be extended to two dimensions. Only one has to choose well the meshes, specifically for the nonstructural ones. 


\section{REFERENCES}

[1] Anaya V., Bendahmane M. Mora D. and Ruiz-Baier R., On a primal-mixed, vorticity-based formulation for reaction-diffusion-Brinkman systems, Submitted 2016.

[2] Anaya V., Bendahmane M. and Sepúlveda M., A numerical analysis of a reaction-diffusion system modelling the dynamics of growth tumors, Math. Models Methods Appl. Sci., 2010; 20(5): 731-756.

[3] Anaya V., Bendahmane M. and Sepúlveda M., Numerical analysis for a three interacting species model with nonlocal and cross diffusion, ESAIM Math. Model. Numer. Anal., 2015; 49(1): 171-192.

[4] Anaya V, Gatica G.N., D. Mora, and Ruiz-Baier R., An augmented velocity-vorticity-pressure formulation for the Brinkman equations, Int. J. Numer. Methods Fluids, 2015; 79 (3): 109-137.

[5] Anaya V., D. Mora D., Oyarzúa R., and Ruiz-Baier R., A priori and a posteriori error analysis of a fully-mixed scheme for the Brinkman problem, Numer. Math., 2016; 133(4): 781-817.

[6] Andreianov B., Bendahmane M. and Ruiz-Baier R., Analysis of a finite volume method for a cross-diffusion model in population dynamics, Math. Models Methods Appl. Sci., 2011; 21(2): 307-344.

[7] Banasiak J. and Lachowicz M., Methods of Small Parameter in Mathematical Biology, Modeling and Simulation in Science, Engineering and Technology Birkhäuser, 2014.

[8] Barrett J. W. and Blowey J. F., Finite element approximation of a nonlinear cross-diffusion population model, Numer. Math., 2004; 98: 195-221.

[9] Bellomo N., Bellouquid A. and Chouhad N., From a multiscale derivation of nonlinear cross-diffusion models to Keller-Segel models in a Navier-Stokes fluid, Math. Models Methods Appl. Sci., 2016; 26: $2041-2069$.

[10] Bellomo N., Bellouquid A., Nieto J. and Soler J., Multiscale biological tissue models and flux-limited chemotaxis for multicellular growing systems, Math. Models Methods Appl. Sci., 2010; 20 : 1179-1207.

[11] Bellomo N., Bellouquid A., Nieto J. and Soler J., On the asymptotic theory from microscopic to macroscopic growing tissue models: An overview with perspectives, Math. Models Methods Appl. Sci., 2012; $22(1)$ : 1130001.

[12] Bellomo N., Bellouquid A., Tao Y. and Winkler M., Toward a mathematical theory of Keller-Segel models of pattern formation in biological tissues, Math. Models Methods Appl. Sci., 2015; 25: 1663-1763.

[13] Bellouquid A., On the asymptotic analysis of the BGK model toward the incompressible linear Navier-Stokes equation, Math. Models Methods Appl. Sci., 2010; 20: 1299-1318.

[14] Bellouquid A. and Tagoudjeu J., An asymptotic preserving scheme for kinetic models for chemotaxis phenomena, arXiv preprint, 2015; arXiv: 1507.05182.

[15] Bendahmane M., Weak and classical solutions to predator-prey system with cross-diffusion, Nonlinear Analysis, $2010 ; 73(8): 2489-2503$.

[16] M. Bendahmane and M. Langlais, A reaction-diffusion system with cross-diffusion modelling the spread of an epidemic disease, Journal of Evolution Equations, 2010; 10(4): 883-904.

[17] Bennoune M., Lemou M. and Mieussens L., An asymptotic preserving scheme for the Kac model of the Boltzmann equation in the diffusion limit, Continuum Mech. Thermodyn., 2009; 21: 401-421.

[18] Burini D. and Chouhad N., Hilbert method toward a multiscale analysis from kinetic to macroscopic models for active particles Math. Models Methods Appl. Sci., 2017; 27(7): 2041-2069.

[19] Carrillo J. A. and Yan B., An Asymptotic Preserving Scheme for the Diffusive Limit of Kinetic systems for Chemotaxis, Multiscale Model. Simul., 2013; 11(1): 336-361.

[20] Chalub F. A., Markowich P., Perthame B., Schmeiser C., Kinetic Models for Chemotaxis and their DriftDiffusion Limits, Monatsh. Math., 2004; 142: 123-141.

[21] Chen L. and Jüngel A., Analysis of a multi-dimensional parabolic population model with strong cross diffusion, SIAM J. Math. Anal., 2004; 36: 301-322.

[22] Chen L. and Jüngel A., Analysis of a parabolic cross-diffusion population model without self-diffusion, J. Diff. Eqns., 2006; 224: 39-59.

[23] Crouseilles n., Hivert H. and Lemou M., Numerical Schemes for Kinetic Equations in the Anomalous Diffusion Limit. Part II: Degenerate Collision Frequency, SIAM J. Sci. Comput., 2016; 38(4):A2464-A2491.

[24] Crouseilles n., Hivert H. and Lemou M., Numerical Schemes for Kinetic Equations in the Anomalous Diffusion Limit. Part I: The Case of Heavy-Tailed Equilibrium, SIAM J. Sci. Comput., 2016; 38(2): A737-A764.

[25] Crouseilles N. and Lemou M., An asymptotic preserving scheme based on a micro-macro decomposition for collisional Vlasov equations: diffusion and high-field scaling limits, Kinetic Related Models, 2011; 4: $441-477$.

[26] Eymard R., Gallout Th. and Herbin R., Finite volume methods.In: Handbook of Numerical Analysis, vol. VII, North-Holland, Amsterdam, 2000.

[27] Gatica G.N., A Simple Introduction to the Mixed Finite Element Method. Theory and Applications, Springer, Briefs in Mathematics, Springer, Cham Heidelberg New York Dordrecht London, 2014.

[28] Golse F., Jin S. and Levermore C. D., A domain decomposition analysis for a two-scale linear transport problem, M2AN Math. Model. Numer. Anal., 2003; 37(6): 869-892.

[29] Gambino G., Lombardo M.C . and Sammartino M., A velocity-diffusion method for a Lotka-Volterra system with nonlinear cross and self-diffusion, Appl. Num. Math., 2009; 59: 1059-1074.

[30] Hillen T. and Othmer H.G., The diffusion limit of transport equations derived from velocity jump processes, SIAM J. Appl. Math., 2000; 61: 751-775.

[31] Jügl A., Diffusive and nondiffusive population models, in Mathematical Modeling of Collective Behavior in Socio-Economic and Life Sciences, eds. G. Naldi, L. Pareschi and G. Toscani, Birkhäuser, 2010.

[32] Keller E.F. and Segel L.A., The Keller-Segel model of chemotaxis, J Theor Biol. 1970; 26: 399-415. 
[33] Klar A., Asymptotic-induced domain decomposition methods for kinetic and drift diffusion semiconductor equations, SIAM J. Sci. Comput., 1998; 19(6): 2032-2050.

[34] Lemou M. and Mieussens L., A new asymptotic preserving scheme based on micro-macro formulation for linear kinetic equations in the diffusion limit, SIAM J. Sci. Comput., 2008; 31(1):334-368.

[35] Lenarda P., Paggi M. and Ruiz Baier R., Partitioned coupling of advection-diffusion-reaction systems and Brinkman flows, J. Comput. Phys., 2017; 344: 281-302.

[36] Okubo A., Diffusion and Ecological Problems: Mathematical Models, Biomathematics, 1980; vol. 10. Springer, Berlin.

[37] Othmer H.G. and Hillen T., The diffusion limit of transport equations II: chemotaxis equations, SIAM J. Appl. Math., 2002; 62: 1222-1250.

[38] Outada N., Vauchelet N., Akrid T. and Khaladi M., From kinetic theory of multicellular systems to hyperbolic tissue equations: Asymptotic limits and computing, Math. Models Methods Appl. Sci., 2016; 62: $2709-2734$.

[39] Simon J., Compact sets in the space $L^{p}(0 ; T ; B)$, Ann. Mat. Pura Appl., 1987; 4: 65-96.

[40] Shigesada N. and Kawasaki K., Biological Invasions: Theory and Practice, Oxford University Press; 1997.

[41] Tian C., Lin Z. and Pedersen M., Instability induced by cross-diffusion in reaction-diffusion systems, Nonl. Anal.: Real World Appl., 2010; 11: 1036-1045.

[42] Vassilevski P.S. and Villa U., A mixed formulation for the Brinkman problem, SIAM J. Numer. Anal., 2014; 52(1): 258-281.

[43] Wen Z. and Fu S., Global solutions to a class of multi-species reaction-diffusion systems with cross-diffusions arising in population dynamics, J. Comp. Appl. Math., 2009; 230: 34-43.

Received December 19, 2017; revised xxxx 20xx.

E-mail address: mostafa.bendahmane@u-bordeaux.fr

E-mail address: fa.karami@uca.ma

E-mail address: zagourmohamed@gmail.com 


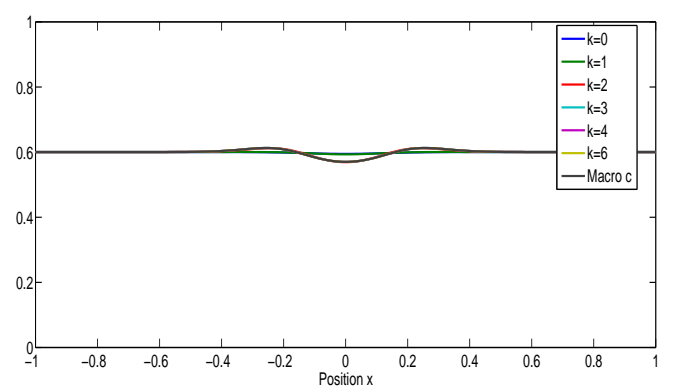

(a)

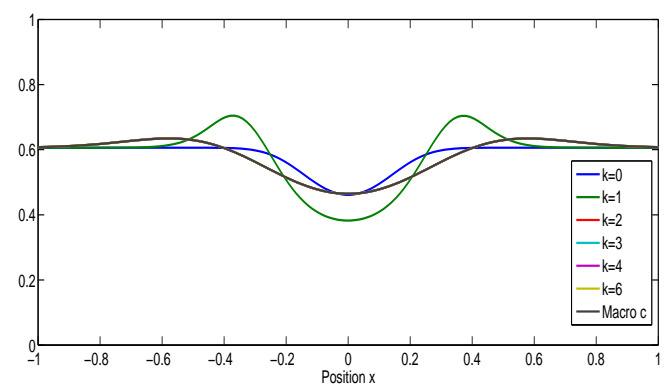

(c)

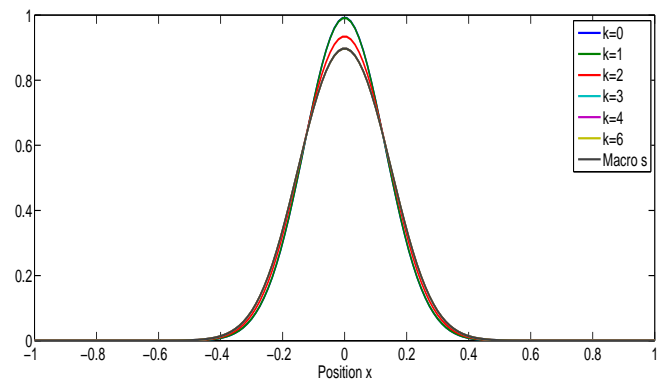

(e)

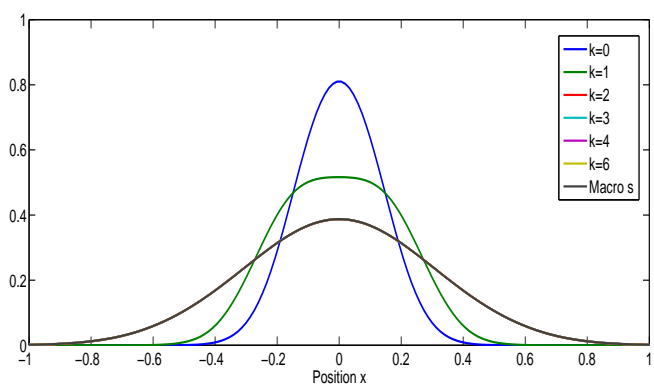

(g)

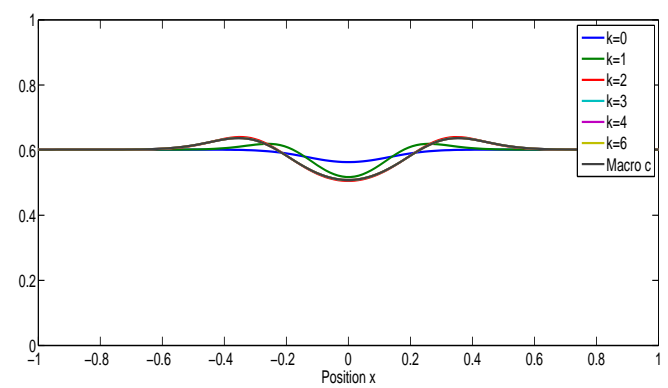

(b)

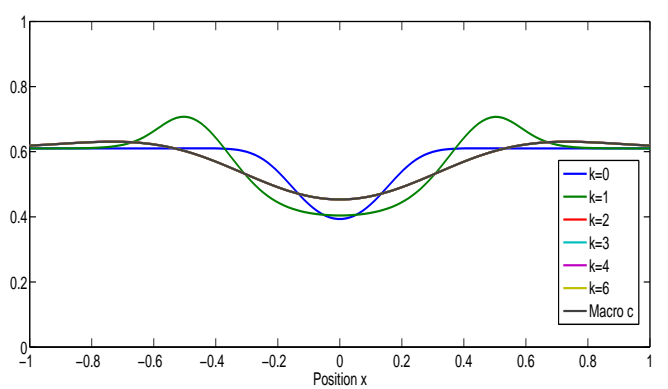

(d)

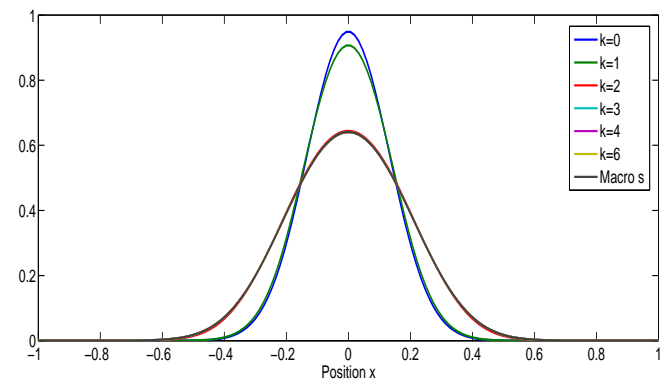

(f)

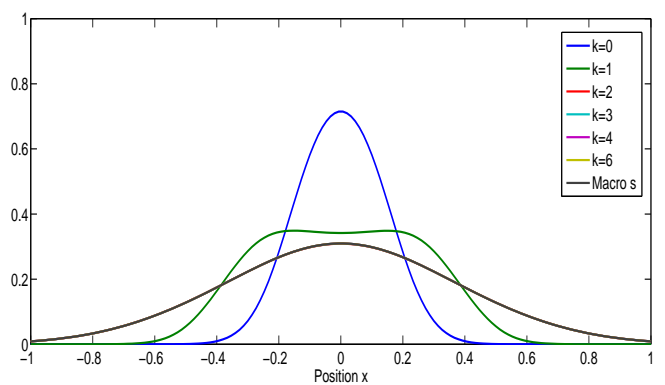

(h)

Figure 2. The Subfigures (a), (b), (c), (d) present time dynamics of predators densities $c(t ; x)$, while Subfigures $(\mathrm{e}),(\mathrm{f}),(\mathrm{g}),(\mathrm{h})$ present time dynamics of preys densities $s(t ; x)$ at $t=0.02,0.04,0.07,0.1$ obtained from the AP scheme with $\varepsilon=10^{-k}, k=0 ; 1 ; 2 ; 3 ; 6$ and comparison with cross-diffusion-Brinkman model on the domain $[-1 ; 1]$ and initial conditions are given by $c_{0}=0.65$ and $s_{0}=\exp \left(30 x^{2}\right)$ in the case: $u=0$. 


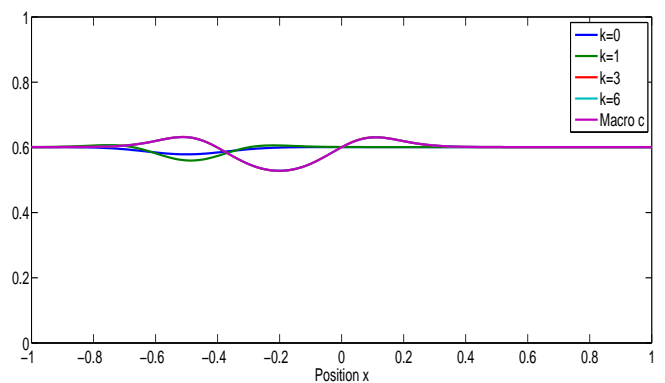

(a)

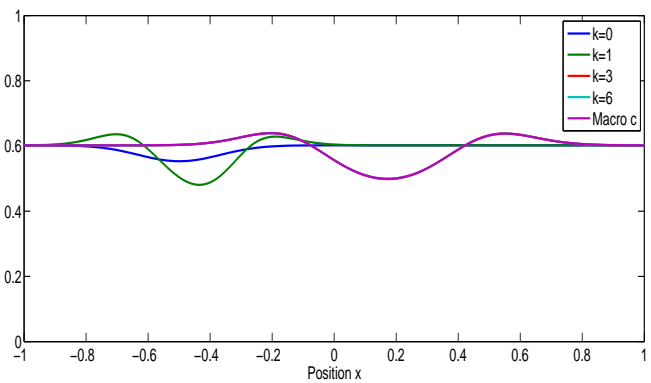

(c)

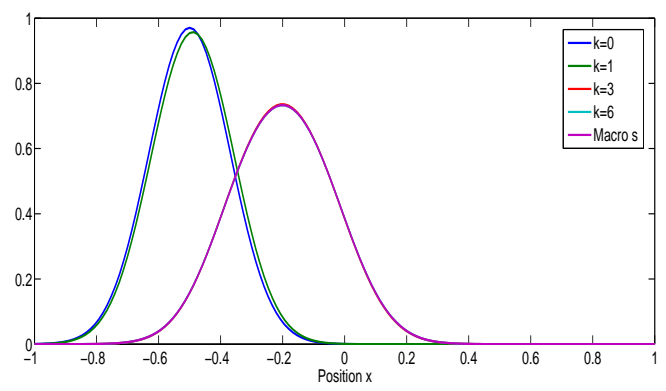

(e)

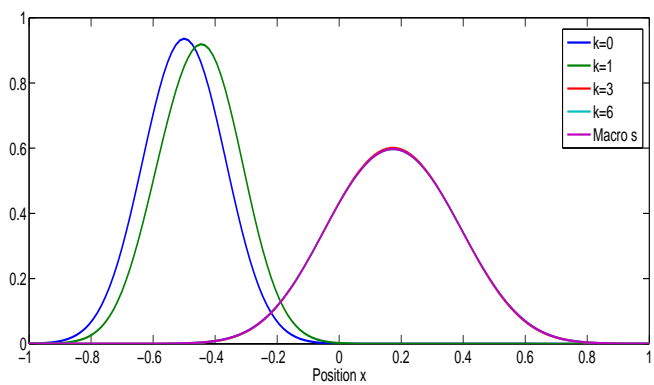

(g)

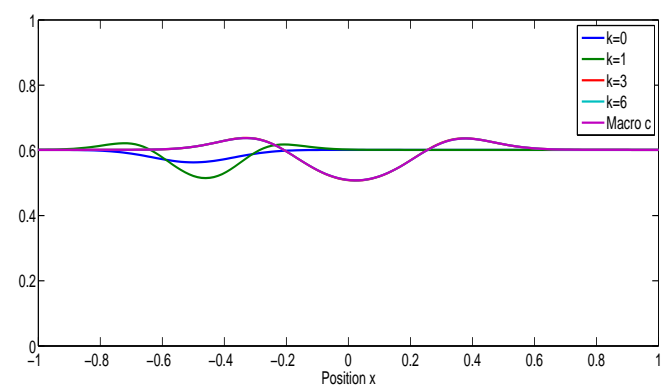

(b)

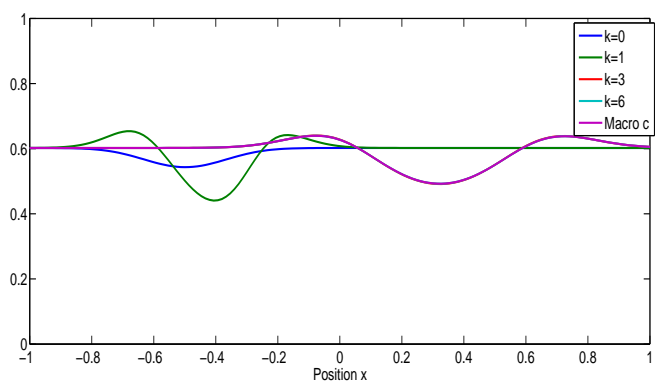

(d)

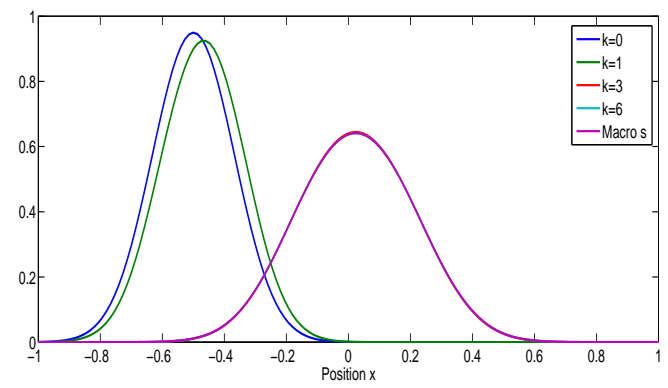

(f)

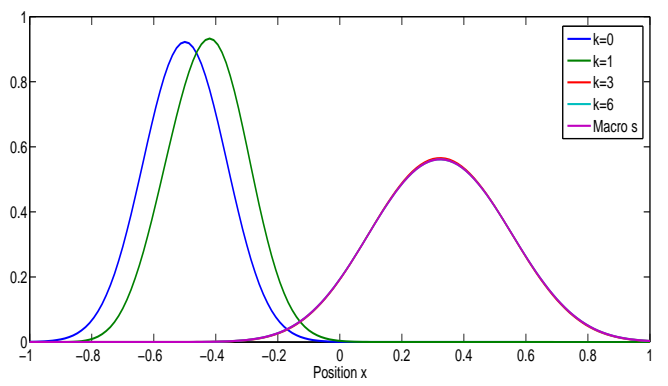

(h)

Figure 3. The Subfigures (a), (b), (c), (d) present time dynamics of predators densities $c(t ; x)$, while Subfigures $(\mathrm{e}),(\mathrm{f}),(\mathrm{g}),(\mathrm{h})$ present time dynamics of preys densities $s(t ; x)$ at $t=0.02,0.04,0.07,0.1$ obtained from the AP scheme with $\varepsilon=10^{-k}, k=0 ; 1 ; 2 ; 3 ; 6$ and comparison with cross-diffusion-Brinkman model on the domain $[-1 ; 1]$ and initial conditions are given by $c_{0}=0.65$ and $s_{0}=$ $\exp \left(30(x+0.5)^{2}\right)$ in the case: $u=1$. 


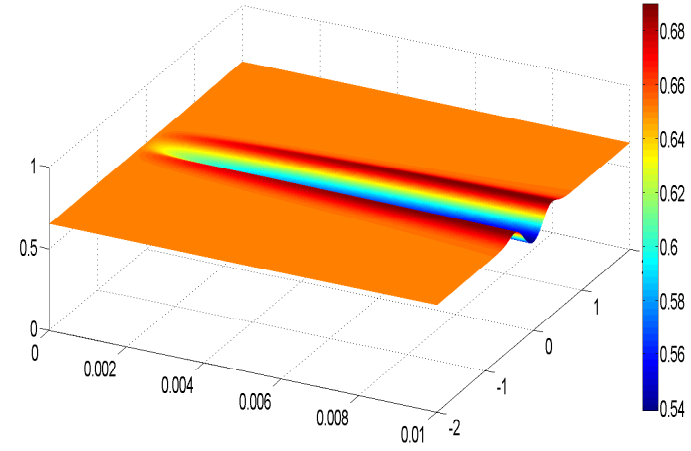

(a)

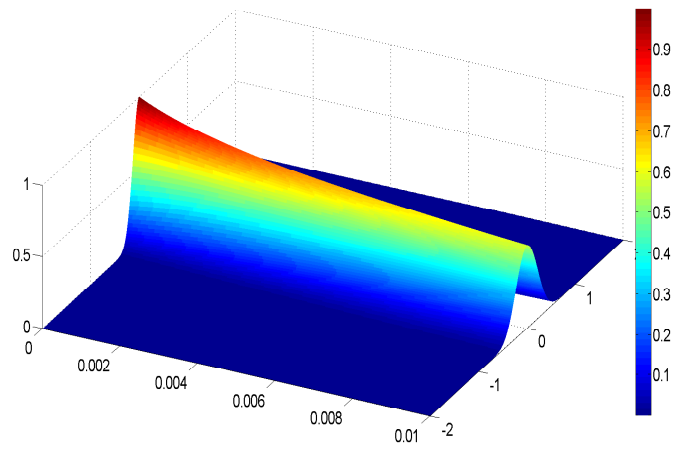

(b)

Figure 4 . Evolution of the densities $c(t ; x)$ and $s(t ; x)$ using micro-macro scheme for $\varepsilon=10^{-6}$ in the case $u=0$.

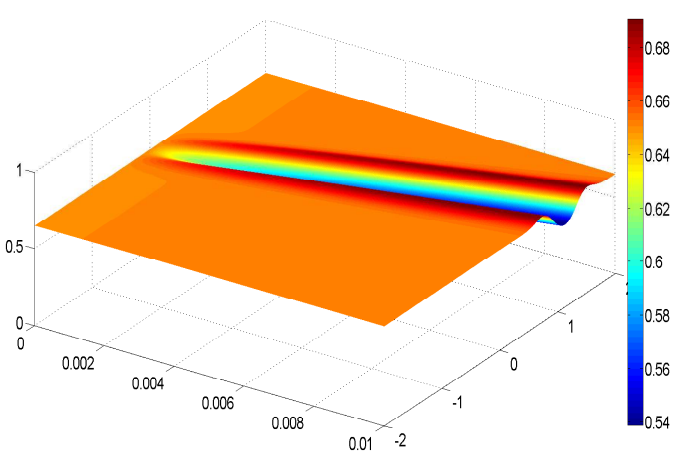

(a)

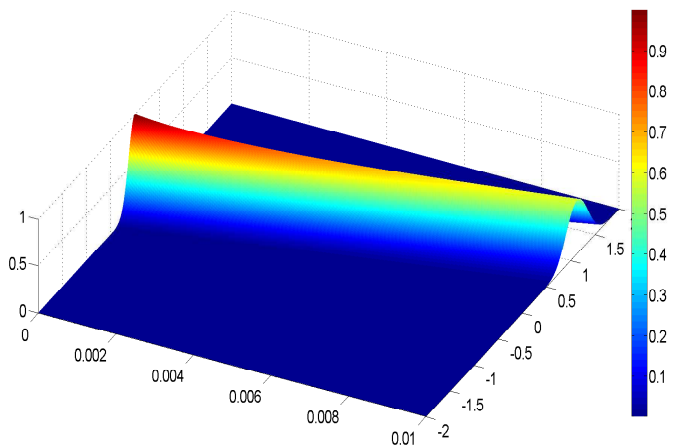

(b)

Figure 5. Evolution of the densities $c(t ; x)$ and $s(t ; x)$ using micro-macro scheme for $\varepsilon=10^{-6}$ in the case $u=1$.

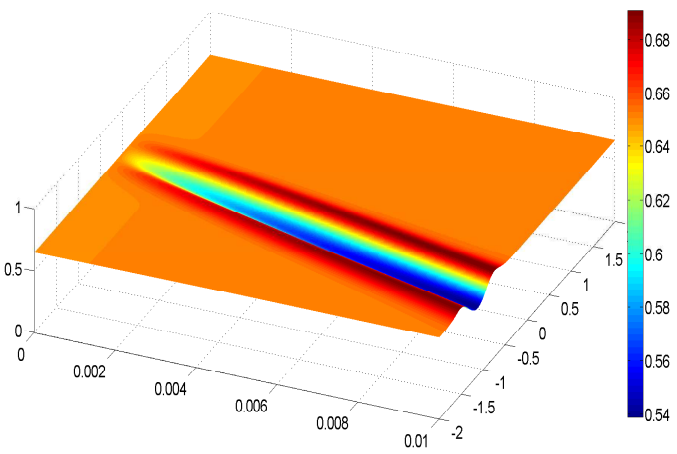

(a)

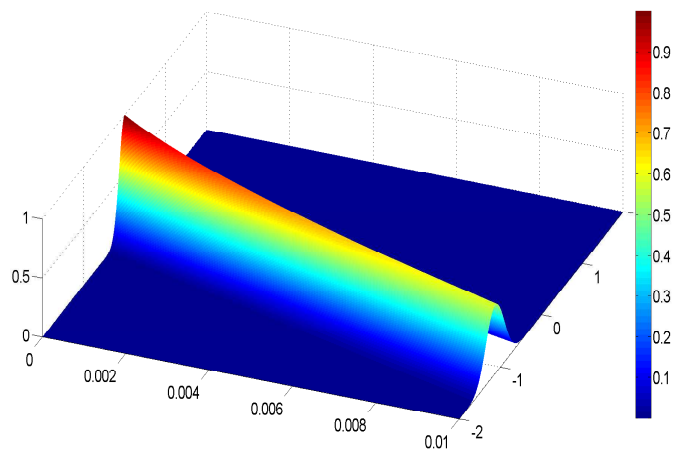

(b)

Figure 6. Evolution of the densities $c(t ; x)$ and $s(t ; x)$ using micro-macro scheme for $\varepsilon=10^{-6}$ in the case $u=-1$. 